\title{
Cloud feedback studies with a physics grid
}

\author{
February 7, 2013
}

Preface: This project was funded by DOE in 2007 but work was initially delayed, first because a senior investigator on the original project (Stephen Thomas) took another position, and subsequently because the PI changed institutions, leaving UCLA in the summer of 2008 to join the MPI-M in Hamburg Germany. The project was moved to the MPI-M with the help of DOE staff, and after negotiating numerous logistical and administrative hurdles the project started in April 2010. Due to differences in funding the project funding was able to support roughly three person years, and our institution also agreed to supplement the support to fund the main postdoc for a third year.

Due to the change of institutions, and considerable development in dynamical cores since the initiation of the project it was decided to work through the ideas using the new ICON dynamical core. The ICON dynamical core is based on a icosahedral grid and serves a prototype for dynamical cores that are being developed in a number of institutions, including at NCAR (the so called MPAS model) for a future version of the Community Earth System Model. The advantage of using ICON for these purposes is that the the ICON dynamical core is relatively stable, and is built on a hierarchical concept that naturally adapts itself to the proposed experimental strategy. Using ICON also allows the project to benefit from staff at the institute who have helped develop ICON, thus bring the main ideas more rapidly to fruition. However, because of the on-going development in the very core of the model and other issues related to pre and post processing, the project did not end in the expected manner. Although the plans in the proposed project has been achieved, to run the heterogeneous multiscale model at global scale requires very heavy computing resources which was not expected. We have found some encouraging results using the available resources on a smaller domain and we plan to investigate them further on a larger scale in near future.

\section{Progress report}

The project timetable in the proposal had identified following yearly milestone:

Year 1: Workshop at NCAR to refine and fix strategy and specify specific design requirements of the physics grid. Funding is included to support 
outside participants, which hopefully will provide a basis for incorporating the views and expertise of scientists at national labs working on related issues. Milestone:Development and initial aqua-planet tests of a physics grid with only horizontal remapping using a modular interpolation scheme that allows tests under different re-mappings.

Year 2: Finalization of aqua-planet convergence studies with traditional parameterization (generalized Williamson (1999) experiments). Introduction of vertical refinement and extension of convergence studies to allow for vertical resolution dependence. Work with atmospheric model working group in the context of identifying and articulating known vertical grid dependencies in existing parameterization. Milestone:Submission of paper describing horizontal remapping and convergence study results.

Year 3: Development of interface for non-uniform distribution of largeeddy simulation as micro-solver. Extension of year 1 and 2 work to irregular physical grid geometries, thereby allowing coupling with surface or subsurface (ocean/sea-ice) models. Aqua-planet tests with UCLA-LES (Stevens et al., 1999) as the micro-solver at specific latitudes. Milestone: Ability and experience running the CAM using the (Heterogeneous Multiscale Method) HMM with the UCLA-LES as the micro-scale solver (at place).

Year 4: Cloud feedback studies using HMM integrations of CAM with the UCLA-LES embedded at specific latitudes identified as key to the cloud feedback from shallow convection in the aqua-planet. Convergence studies both as a function of micro and macro-scale grids. Milestone:Submission of paper bounding cloud feedbacks from the zonally symmetric contribution of shallow convection. It is anticipated that this will be a high-profile paper which provides the first rational (numerically, and physically sound) estimates of cloud feedbacks from a critically important cloud regime.

Since the submission of the first report which covered the milestones of Year 1 and 2, further changes have been made in the model to incorporate the UCLALES into the global model to perform heterogeneous multiscale modeling. As mentioned in the Year 4 plan above, cloud feedback studies have also been performed in the aqua-planet setup but not for the whole globe. Due to unavailability of computational resources and due the developing nature of the new model ICON, we have made cloud feedback studies only for a smaller domain. We anticipate global runs in near future.

This report has been organized in two parts. The first part is mostly from the first report summarizing the achievements made in the first 18 months of the project. The second part focuses on the cloud feedback studies using heterogeneous multiscale model that summarizes the achievements of the next 18 months. 


\section{Contents}

1 Progress report 1

I First part- the dual-grid approach 4

2 Dual-grid approach $\quad 4$

2.1 Conservative interpolation . . . . . . . . . . . . 5

3 Integration of physics grid into ICON $\quad 7$

4 Test case and discussions 9

4.1 Initial conditions . . . . . . . . . . . . . . . . . . 9

4.2 Results and discussions . . . . . . . . . . . . . 10

II Development of Heterogeneous Multiscale Modeling Kernel in ICON

5 Heterogeneous Multiscale Modeling $\quad 18$

6 Evaluating classical parameterization 20

6.1 Large-scale forcing and Time-varying statistics . . . . . . . . . . 23

6.2 Liquid water potential temperature budget . . . . . . . . . . 26

6.3 Moisture budget . . . . . . . . . . . . . . . . . . . 27

6.4 Cloud water budget . . . . . . . . . . . . . . . . 29

$\begin{array}{lll}7 & \text { Summary } & 32\end{array}$ 


\section{Part I}

\section{First part- the dual-grid approach}

\section{Dual-grid approach}

Idea behind dual-grid approach is to have a physics grid independent of the dynamics grid for parameterization. As a first step, we keep the vertical resolution same in both the dynamics and the physics grid to follow the setup of Williamson (1999). The key question is where we should we keep the horizontal resolution of the physics grid, $\Delta_{p}$, coarser or finer than the dynamic grid resolution, $\Delta_{d}$. To answer that question, we followed the arguments of Lander and Hoskins (1997) in which it was proposed to use a coarser grid for parameterization. From their analysis they found that the half-width of the smallest believable local feature is about 1.6 times the half-wavelength associated with the truncation scale, and the remaining small scale features are unbelievable. They further write that "the aim must be to provide input to the gridpoint parameterization schemes that is reliable and for these schemes to produce output for the dynamical model on scales with which the dynamical model can cope".

Because their finding was based on the use of a spectral model, we suspect the smallest believable scale in our second-order spatial finite-volume discretization scheme to be even larger. This becomes obvious when we see the spectral accuracy of a second-order method in Fig. 1. The figure clearly shows that the smallest believable scale in a second-order method is about $(1 / 12)^{t h}$ of that captured by a spectral method. Without going into further details to quantify the believable scales in a finite-volume method, we assume at this point that the physics grid size, $\Delta_{p}$, acts as a cut-off between the believable (or reliable) and the unbelievable scales in the dual-grid approach. Therefore, we assume that only the waves with wavelength larger than $2 \Delta_{p}$ are accurately represented in the dynamics grid, and hence only those scales are forced with the output from a parameterization scheme.

If we write $T_{d}$ as the temperature on the high resolution dynamics grid, and $T_{p}$ as the temperature on the coarse resolution physics grid, then the algorithm for the dual-grid approach can be expressed as:

- Compute the tendency $\delta T_{d}$ due to dynamics alone.

- Interpolate the dynamics tendency to the physics grid to get $\left(\delta T_{d}\right)_{\Delta_{p}}$.

- Interpolate the state variables required by the physics package using the same method.

- Compute tendency $\delta T_{p}$ due to the physical processes alone.

- Calculate net tendency, dynamical plus physical as $\delta T=\delta T_{d}+\delta T_{p}$. Because $\delta T_{p}$ is calculated on coarser physics grid, the sum ensures that only scales of the order of $\Delta_{p}$ are forced in $\delta T_{d}$ 


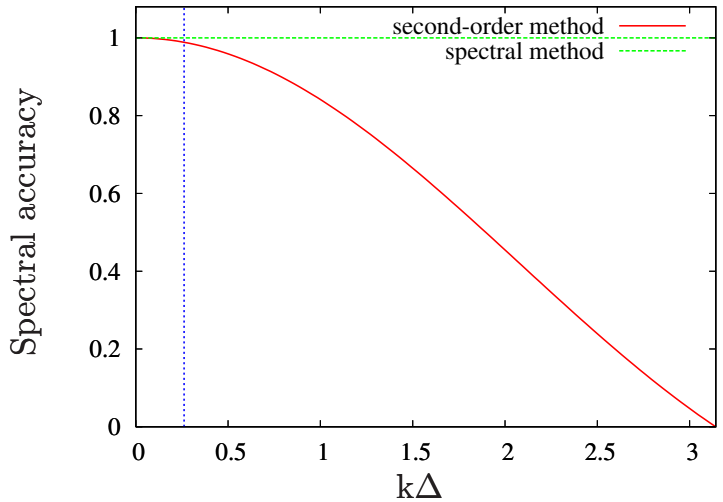

Figure 1: Spectral accuracy of second-order central difference scheme. The vertical line indicates the location $(\mathrm{k} \Delta=\pi / 10)$ where it departs from a spectral method. $k$ in the figure is the spectral wave number and $\Delta$ is grid size.

- Integrate the dynamics state in time to get $T_{d}^{n+1}=T_{d}^{n}+\delta T$.

This algorithm does not require any hampering of the physics package and is therefore easy to implement. However, the GridAlt algorithm of Molod (2009) is a good alternative in the sense that it avoids the interpolation step 3 altogether. There are other issues in the GridAlt algorithm which we plan to deal with in the coming days. The important step in the above algorithm is interpolation from dynamics-to-physics grid which is discussed next. For an interpolation scheme, apart from accuracy, the most desirable property is that it should not create any spurious source or sink of global invariant quantities like mass and energy. This is achieved in the present approach with the use of a conservative interpolation technique.

\subsection{Conservative interpolation}

The two commonly used interpolation techniques are: (a) conservative interpolation based on finite-volume techniques, and (b) functional interpolations (such as Lagrange interpolations), which are usually non-conservative. Molod (2009) used a non-conservative interpolation in a way that the dynamics-tophysics interpolation is exactly reversed with a physics-to-dynamics interpolation. Whereas, Vintzileos and Sadourny (1997) in their delocalized physics approach used an interpolation scheme that conserved the fluxes while going from dynamics-to-physics grid, at the same time ensuring that the same scheme is reversed when integrating back to the dynamics grid. It is now widely accepted that for long time integration it is favorable to use a conservative interpolation (see Dukowicz and Kodis (1987); Jones (1999); Lauritzen and Nair (2008)), but it is not clear conservation of which quantity gives better result. We have performed interpolation conserving mass, momentum, enthalpy, and total tracer. 
Details are provided below after explaining the working of a general conservative interpolation method as explained in Jones (1999).

Suppose we want to conserve the global integral of a quantity $f$ on a source grid when moving to a destination grid. Let quantity is denoted as $F$ on the destination grid. To ensure conservation, the quantity $F$ at a destination grid cell $k$ must satisfy

$$
\bar{F}_{k}=\frac{1}{A_{k}} \int_{A_{k}} f d A,
$$

where $\bar{F}$ is the area-averaged quantity and $A_{k}$ is the area of cell $k$. Note that the integral is over the area of the destination grid cell, therefore only those grid cells on the source grid that are covered at least partly by the destination grid cell contribute in $F_{k}$. Satisfying Eq. 1 will automatically satisfy the global conservation condition

$$
\int_{A} F d A=\int_{A} f d A,
$$

where the integral is over the entire grid surface $A$. If cell $k$ overlaps $N$ cells on the source grid, the interpolation can be written as

$$
\bar{F}_{k}=\frac{1}{A_{k}} \Sigma_{n=1}^{N} \int_{A_{n} k} f_{n} d A,
$$

where $A_{n k}$ is the area of the source grid cell $n$ covered by the destination grid cell $k$, and $f_{n}$ is the local value of the $f$ in the grid cell $n$.

Assuming $f_{n}$ is constant across a source grid cell leads to the first-order areaweighted scheme. Following the concept used in designing conservative finitevolume schemes, Dukowicz and Kodis (1987) suggested following expansion for $f_{n}$ within a grid cell $n$

$$
f_{n}=\bar{f}_{n}+\nabla_{n} f .\left(\boldsymbol{r}-\boldsymbol{r}_{n}\right) .
$$

Here, $\boldsymbol{\nabla}_{n} f$ is the gradient of $f$ in cell $n$ and $\boldsymbol{r}_{n}$ is the centroid. Clearly, expansion in Eq. 4 satisfies the conservation constraint

$$
\bar{f}_{n}=\frac{1}{A_{n}} \int_{A_{n}} f_{n} d A .
$$

The interpolation is thus second order accurate if $\nabla_{n} f$ is at least a first-order approximation.

The physics package in ICON is designed in a way that it explicitly requires state variables from previous time step and some of the tendencies. We have used different conservation constraints for different state variables as listed below:

mass conservation: Because density is not a prognostic variable in our system of equations, mass conservation is achieved in two steps. First step requires 
calculation of $\bar{F}=\bar{\rho}_{p}$ on the physics grid from $\bar{f}=\bar{\rho}_{d}$ on the dynamics grid using the algorithm described above. Mass conservation is then achieved by calculating pressure as $\bar{P}_{p}=\bar{\rho}_{p} R \bar{T}_{p}$, where $R$ is the gas constant for the moist air and $\bar{T}_{p}$ is temperature on the physics grid as calculated next.

enthalpy conservation: In order to achieve enthalpy conservation, interpolation is performed using $f=\rho_{d} T_{d}$ to give $\bar{F}=(\overline{\rho T})_{p}$. Temperature on the physics grid cell, $\bar{T}_{p}$, is then calculated as

$$
\bar{T}_{p}=\frac{(\overline{\rho T})_{p}}{\bar{\rho}_{p}} .
$$

This way the global enthalpy $\int \rho C_{p} T d A$ is conserved under the assumption that $C_{p} \approx C_{p_{d}}$ for moist air. $C_{p_{d}}$ is the heat capacity of dry air.

momentum conservation: Momentum is conserved by using $f=\rho_{d} v_{d}$ to give $\bar{F}=(\overline{\rho v})_{p}$. Velocity on the physics grid cell, $\bar{v}_{p}$ is then calculated as

$$
\bar{v}_{p}=\frac{(\overline{\rho v})_{p}}{\bar{\rho}_{p}} .
$$

tracer conservation: Like momentum, tracer is conserved by using $f=\rho_{d} \phi_{d}$ to give $\bar{F}=(\overline{\rho \phi})_{p}$ for any tracer $\phi$. Tracer value at the physics grid cell is then obtained as $\bar{\phi}_{p}=\frac{(\overline{\rho \phi})_{p}}{\bar{\rho}_{p}}$.

It makes sense to interpolate the tendencies using the same strategy to consistently conserve mass, enthalpy, momentum, and total tracer during the interpolation.

\section{Integration of physics grid into ICON}

ICON (ICOsahedral Non-hydrostatic General Circulation Model) is a new development initiated by the Max Planck Institute for Meteorology (MPI-M) and the Deutscher Wetterdienst (DWD). This finite-volume dynamical core combines several properties considered important for future progress in numerical weather forecasting and climate research. It uses the geodesic Delaunay grids with C-type staggering with an inbuilt ability to perform limited area simulation. For the present report it suffices to state that the mass points are located at the centers of the Delaunay (triangular) grid cells, and the velocity points are located at the mid-point of the edges of the triangular cells. By construction these mid-points are the points of intersection of the edges of the Delaunay and Vornoi cells. More on the details of the ICON dynamical core can be found in Bonaventura and Ringler (2005) and the references listed on the website http://www.icon.enes.org/.

The basic method of generating a triangular grid from an icosahedron involves an initial root division of the spherical icosahedron in $n_{r}$ sections followed 
by $n_{b}$ bisections of the triangle edges (Wan, 2009). Resulting mesh is identified as $\mathrm{R} n_{r} \mathrm{~B} n_{b}$. The total number of triangle cells, edges and vertices relate to $n_{r}$ and $n_{b}$ as

$$
\begin{aligned}
& n_{c}=20 n_{r}^{2} 4^{n_{b}}, \\
& n_{e}=30 n_{r}^{2} 4^{n_{b}}, \\
& n_{v}=10 n_{r}^{2} 4^{n_{b}}+2 .
\end{aligned}
$$

In order to explain the working of interpolation on ICON grid, we chose R2B3 as the coarse physics grid and R2B4 as the fine dynamics grid. Grid refinement from B3 to B4 implies that each physics cell now has 4 dynamics cell within (see Fig. 2). Because the destination cell here exactly cover the source grid cells, its not difficult to note that the Eq. 4 on source grid cells reduces to $f_{n}=\bar{f}_{n}$. This implies that the interpolation formula in Eq. 3 reduces to a first order area-weighted interpolation method. While it is possible to get a higher order method following Lauritzen and Nair (2008), we have decided to stay with the first-order method for the time being. Therefore, for $N$ dynamic grid cells within a physics grid cells the interpolation formula in Eq. 3 simplifies to

$$
\bar{F}_{k}=\frac{1}{A_{k}} \Sigma_{n=1}^{N} A_{n k} \bar{f}_{n} .
$$

As the tendencies from the physics package are transferred directly to the dynamic cells without any extrapolation, we have on the dynamics grid cells

$$
\bar{f}_{n k}=\bar{F}_{k} \quad \text { for } \quad n \in[1, N],
$$

for physical tendency $\bar{F}_{k}$ calculated on the $k^{\text {th }}$ grid cell.

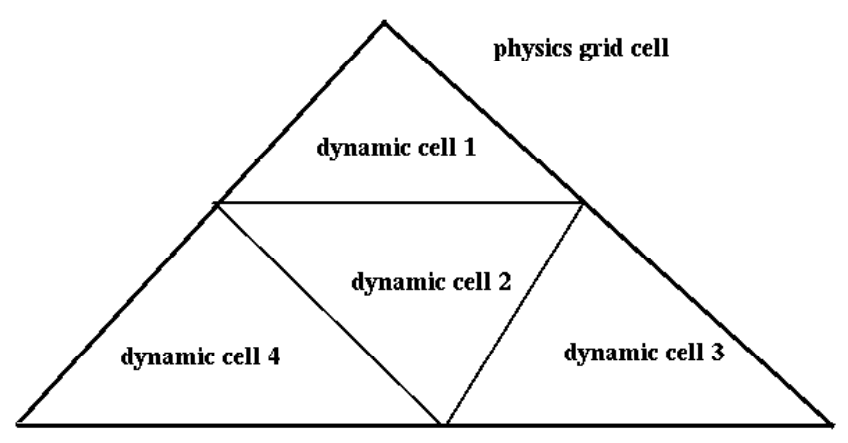

Figure 2: Schematic showing four dynamic-grid cells in a physics grid cell 


\section{Test case and discussions}

To check the correctness of the dual-grid approach in ICON, we ran the Baroclinic wave instability test case of Jablonowski and Williamson (2006) in the atmosphere only mode of ICON. Though the test case is primarily designed for testing the dry dynamical cores, we have run it with these physical processes: (a) vertical diffusion, (b) cumulus convection, and (c) large-scale condensation. The initial conditions for this case are defined by analytical expressions which are a steady state solution of the hydrostatic primitive equations. Baroclinic waves are triggered in the northern hemisphere when the initial solution is overlaid with a perturbation. In general, the baroclinic wave starts growing observably around model day 4 and evolves rapidly thereafter with explosive cyclogenesis at model day 8 . The wave train breaks after day 9 and generates a full circulation in both hemispheres after day 12 .

\subsection{Initial conditions}

Initial conditions are same as in Jablonowski and Williamson (2006). The initial surface pressure is given as $p_{s}=1000 \mathrm{hPa}$ and the initial spherical wind velocities are defined as follows. We describe the initial conditions in horizontal longitudelatitude $(\lambda, \phi)$ coordinates and the vertical coordinate $\eta=\eta\left(p, p_{s}\right)$.

The balanced initial wind velocity $(u, v)$ is given by

$$
\begin{aligned}
& u(\lambda, \phi, \eta)=u_{0} \cos ^{3 / 2} \eta_{v} \sin ^{2}(2 \phi) \\
& v(\lambda, \phi, \eta)=0,
\end{aligned}
$$

where $u_{0}=35 \mathrm{~m} / \mathrm{s}, \eta_{v}=\left(\eta-\eta_{0}\right) \pi / 2, \eta_{0}=0.252$, and $\eta \in[0,1]$. The horizontally averaged temperature $\langle T\rangle(\eta)$ is split into two representations for lower and middle atmosphere as follows:

$$
\begin{aligned}
<T>(\eta) & =T_{0} \eta^{R_{d} \Gamma / g} \quad \text { for } \quad \eta_{s} \geq \eta \geq \eta_{t}, \\
& =T_{0} \eta^{R_{d} \Gamma / g}+\Delta T\left(\eta_{t}-\eta\right)^{5} \quad \text { for } \quad \eta<\eta_{t}
\end{aligned}
$$

where $T_{0}=288 \mathrm{~K}, \Gamma=0.005 \mathrm{~K} / \mathrm{m}, R_{d}=287 \mathrm{~J} / \mathrm{Kg} \mathrm{K}, \mathrm{g}=9.8 \mathrm{~m} / \mathrm{s}^{2}, \Delta T=4.8$ $\times 10^{5} \mathrm{~K}$, and $\eta_{t}=0.2$ is the tropopause level. By defining the following dummy functions:

$$
\begin{aligned}
& A=\left[-2 \sin ^{6} \phi\left(\cos ^{2} \phi+1 / 3\right)+\frac{10}{63}\right], \\
& B=a \Omega\left[\frac{8}{5} \cos ^{3} \phi\left(\sin ^{2} \phi+2 / 3\right)-\frac{\pi}{4}\right],
\end{aligned}
$$

the total temperature is given as 


$$
T(\lambda, \phi, \eta)=<T>(\eta)+\frac{3}{4} \frac{\eta \pi u_{0}}{R_{d}} \sin _{v} \cos ^{1 / 2} \eta_{v} \times\left(2 A u_{0} \cos ^{3 / 2} \eta_{v}+B\right),
$$

where $a=6.371229 \times 10^{6} \mathrm{~m}$ is the earth's radius and $\Omega=7.29212 \times 10^{-5} \mathrm{~s}^{-1}$ is its angular velocity. The surface geopotential is given as

$$
\phi_{s}(\lambda, \phi)=u_{0} \cos ^{3 / 2}\left[\frac{\pi}{2}\left(\eta_{s}-\eta_{0}\right)\right]\left[u_{0} \cos ^{3 / 2}\left(\frac{\pi}{2}\left(\eta_{s}-\eta_{0}\right)\right) A+B\right],
$$

where $\eta_{s}=1$ is the surface level.

The perturbation field added to the zonal wind in Eq. 13 to trigger baroclinic instability is given by

$$
u^{\prime}(\lambda, \phi, \eta)=u_{p} \exp \left[-\left(\frac{r}{R}\right)^{2}\right]
$$

with maximum amplitude $u_{p}=1 \mathrm{~m} / \mathrm{s}$, radius $\mathrm{R}=a / 10$ and the great-circle distance $r$, which is given by

$$
r=a \arccos \left[\sin \phi_{c} \sin \phi+\cos \phi_{c} \cos \phi \cos \left(\lambda-\lambda_{c}\right)\right],
$$

where $\left(\lambda_{c}, \phi_{c}\right)=(\pi / 9,2 \pi / 9)$ is the center of the perturbation geographically located at $\left(20^{\circ} \mathrm{E}, 40^{\circ} \mathrm{N}\right)$.

\subsection{Results and discussions}

The model uses 31 vertical levels till the top of the atmosphere. We ran the model for fixed physics resolution at R2B3, and increasing dynamic resolution. The dual-grid runs are identified as $\mathrm{P} n_{p} \mathrm{D} n_{d}$ where $B=n_{p}$ for the physics grid and $B=n_{d}$ for the dynamics grid. $R$ is fixed at 2 for all the cases discussed. Likewise, the single-grid runs are identified as $\mathrm{P} n_{d-} \mathrm{D} n_{d}$ for $B=n_{d}$ for both the dynamics and the physics grid. Table 1 lists the various cases performed and relevant characteristics of these different grid resolutions. Although not shown, the dual-grid approach reduces the computation time by a factor more than 1.5. It is to be noted that the factor increases with increasing dynamics grid resolution.

At grid resolution R2B3 the characteristic edge length of the physics grid is fixed at $\Delta_{p}=440.87 \mathrm{~km}$, and the characteristic edge length of the dynamics grid is decreased until $\Delta_{d}=55.11 \mathrm{~km}$. To avoid numerical instability, $\Delta t$ for each run is decreased linearly with decreasing $\Delta_{d}$ to maintain a fixed CFL number. The coefficient of the fourth-order artificial diffusion in ICON scales down automatically as $\sim \Delta_{d}^{4}$. This decrease in diffusion coefficient is often scaled to keep the damping of the smallest resolved waves the same (Williamson, 2008). We did not follow this to check how much noise is generated due to interpolation error. 
P3 D4

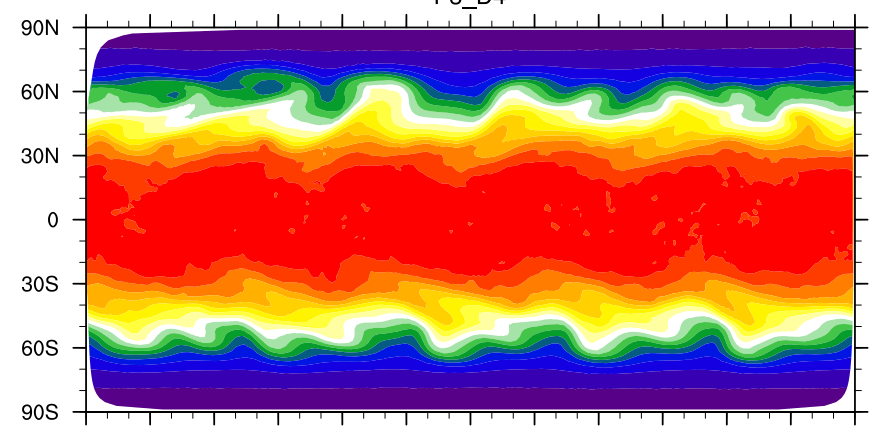

180 150W 120W 90W 60W 30W 0 30E $\quad$ G0E 90 G 120 E 150 E 180

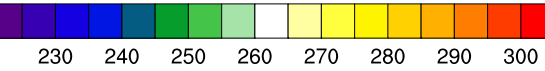

P3_D5

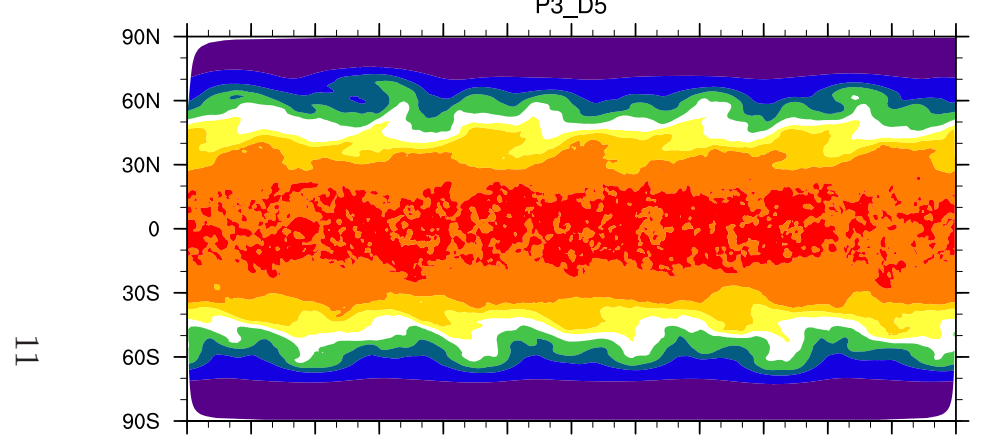

180 150W 120W 90W 6OW 30W 0 30E 60 E $90 \mathrm{E}$ 120E $150 \mathrm{E} \quad 180$

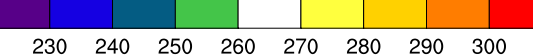

P3 D6

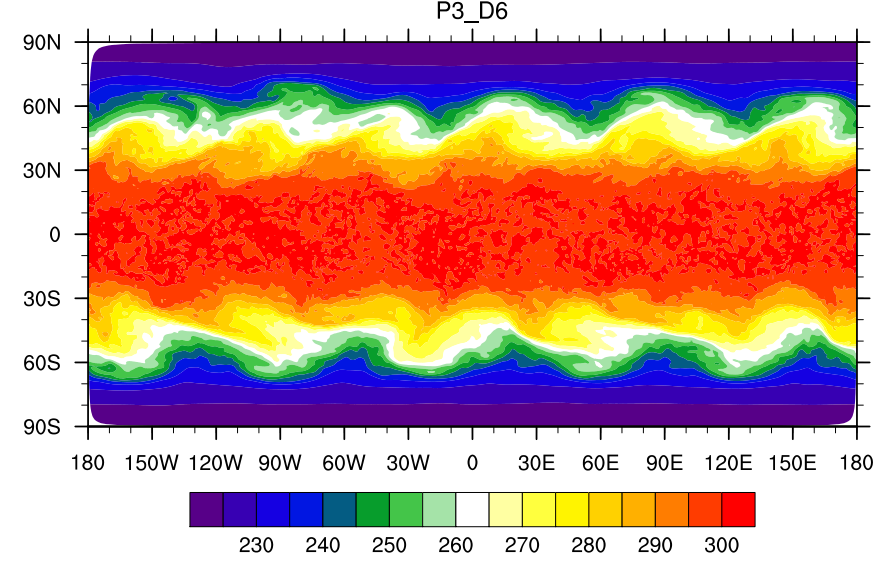

P4_D4

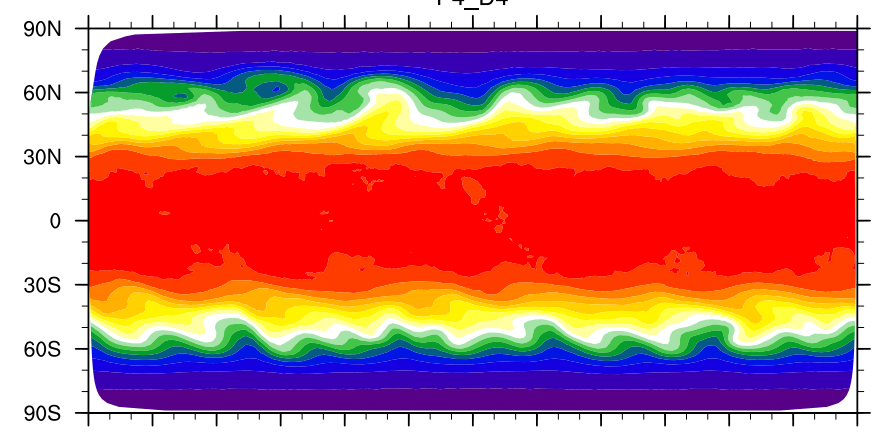

180 150W 120W 90W 60W 30W 0 30E 60 E $90 \mathrm{E}$ 120E $150 \mathrm{E} \quad 180$

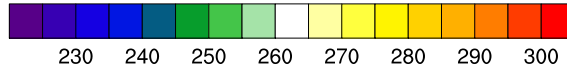

P5_D5

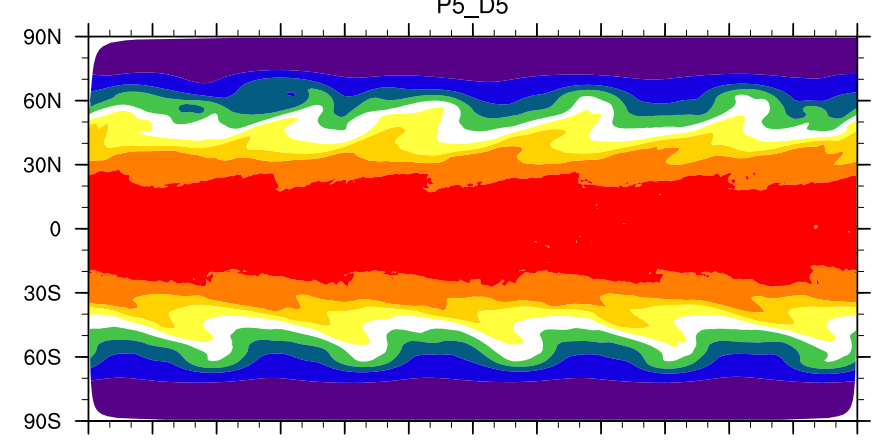

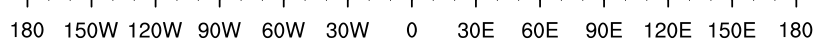

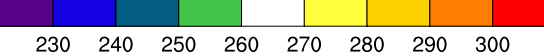

P6 D6

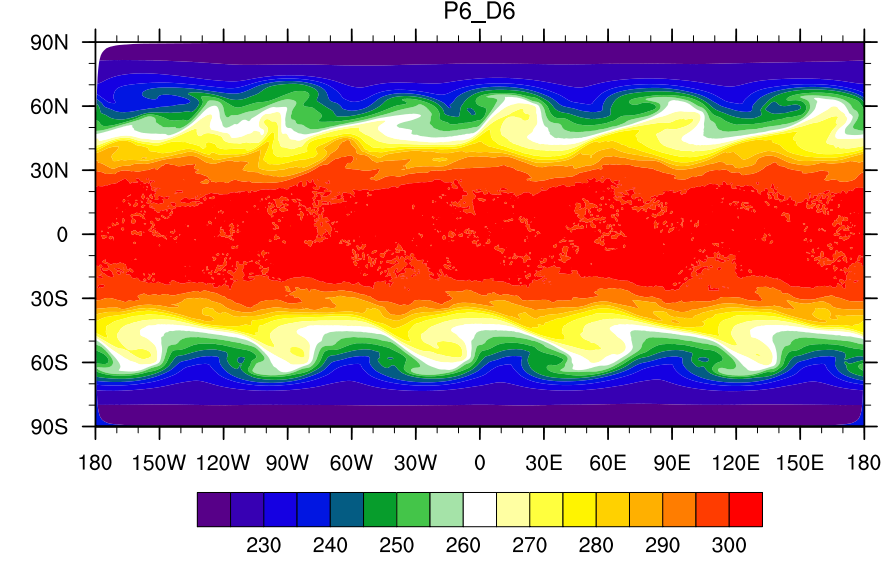

Figure 3: Temperature contours at day 12 of the simulation at an arbitrary $\eta$ level. Left-hand column shows dual-grid results and right-hand column shows the corresponding single-grid results. 
P3 D4

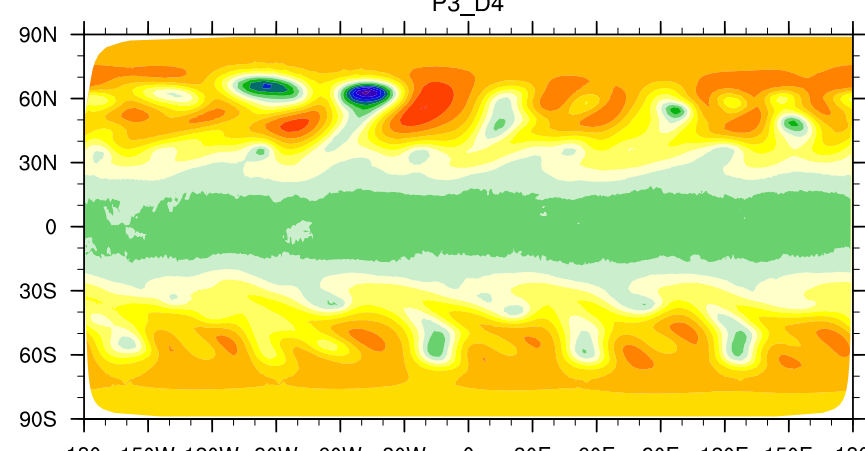

180 150W 120W 90W 6OW 30W O 30 E 60 E 90 E 120 E 150 E 180

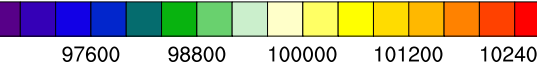

$$
\text { P3_D5 }
$$
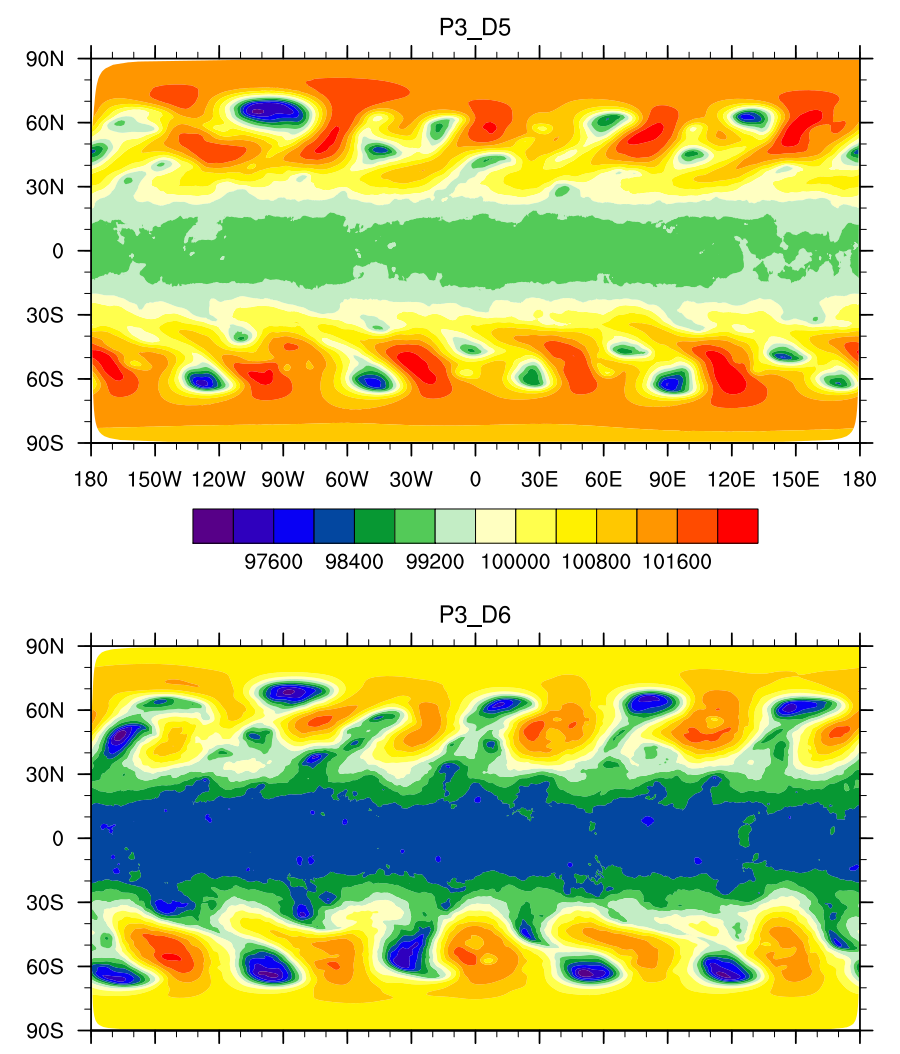

180 150W 120W 90W 60W 30W O 30E 60E 90E 120E 150E 180

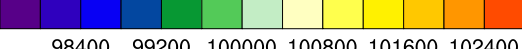

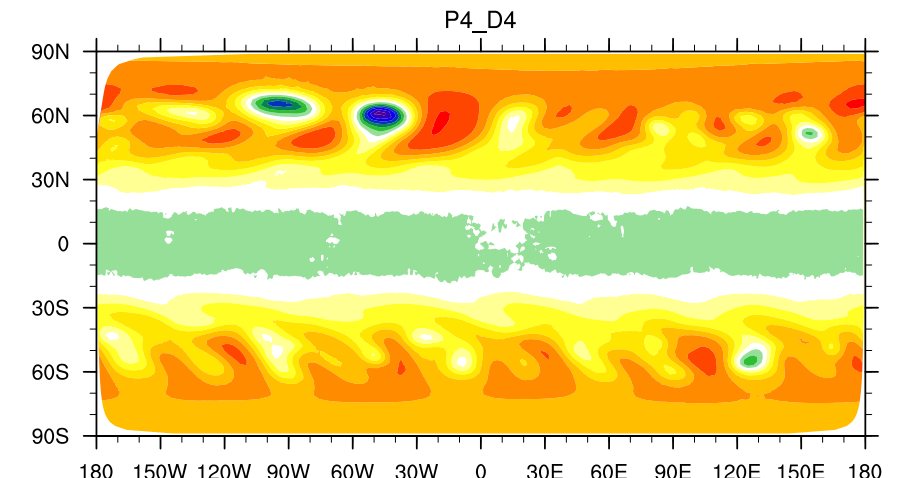

180 150W 120W 90W 6OW 30W 0 30E 60 E 90 E 120 E $150 \mathrm{E} \quad 180$ 96800976009840099200100000100800101600

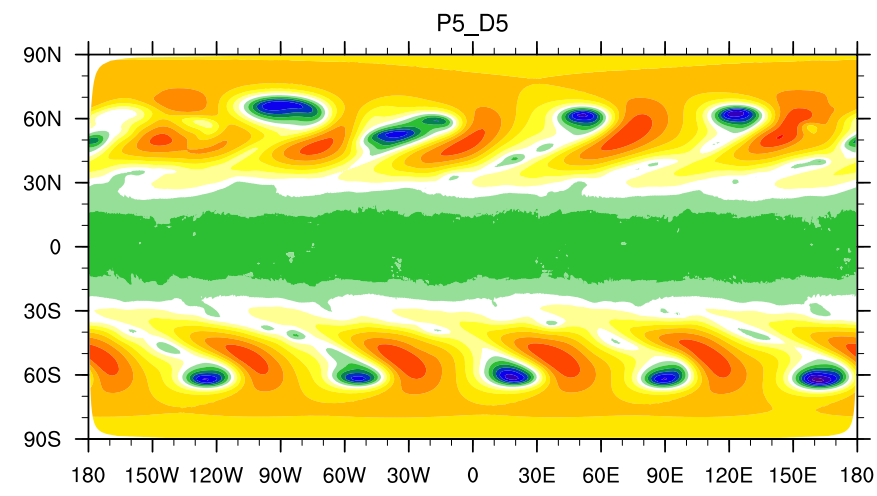

976009840099200100000100800101600102400

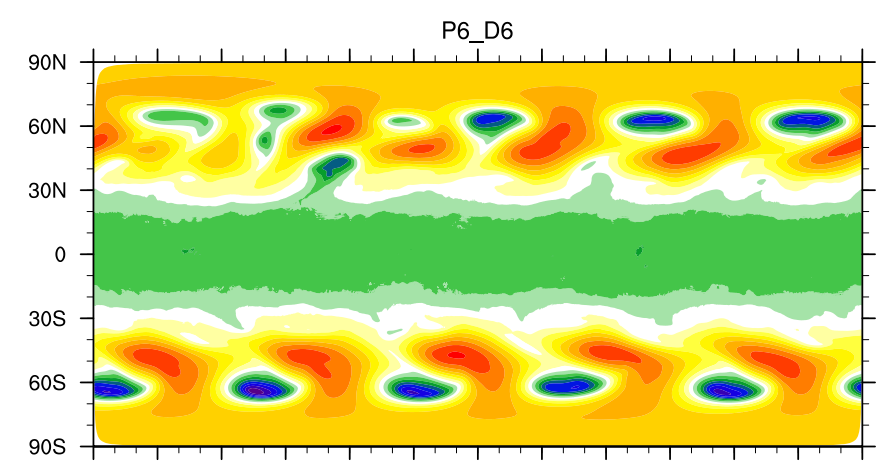

180 150W 120W 90W 60W 30W O 30 E 60E 90E 120 E 150E 180

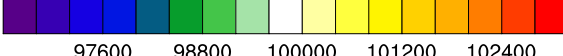

Figure 4: Surface pressure contours at day 12 of the simulation at an arbitrary $\eta$ level. Left-hand column shows dual-grid results and right-hand column shows the corresponding single-grid results. 
Table 1: Single and dual grid simulations at different resolution. The number of triangle cells, the characteristic edge length at each resolution, and time step used in the simulation are also listed.

\begin{tabular}{ccccc}
\hline \hline dual-grid & single-grid & $\begin{array}{c}\text { number of } \\
\text { triangle cells }\end{array}$ & $\begin{array}{c}\text { characteristic edge } \\
\text { length }(\Delta)\end{array}$ & $\Delta t$ \\
\hline P3_D4 & P4_D4 & 20480 & $220.43 \mathrm{~km}$ & $400 \mathrm{sec}$ \\
P3_D5 & P5_D5 & 81920 & $110.22 \mathrm{~km}$ & $200 \mathrm{sec}$ \\
P3_D6 & P6_D6 & 327680 & $55.11 \mathrm{~km}$ & $100 \mathrm{sec}$ \\
\hline
\end{tabular}

Figure 3 compares the temperature contours of dual-grid and single-grid simulations on model day 12 at an arbitrary $\eta$ level. By model day 12 the instability waves are fully developed on both the hemispheres causing breakdown of initial large scale features. This makes the job of interpolation even more challenging. Results in Fig. 3 clearly establish the correctness of the interpolation algorithm. Also, the dual-grid results appear noisy as the resolution is increased. This happens because the applied forcing has potential to create sharp gradient in the total tendency at the forcing scale $\Delta_{p}$. This indicates the need of scaling the diffusion coefficient.

Contrary to our expectation, the dual-grid simulations do not show any convergence in the large scale features for this case. The small scales that are excited due to grid refinement continue to modify the large scale features. For example, the baroclinic wave in the northern hemisphere in P3_D4 shows 5 big troughs with 5 small troughs on its side, whereas the same wave in P3_D6 shows only 5 troughs formed due to the combination of big and small troughs in P3_D4. Same is noted for single-grid results. This suggests a possible convergence at higher resolutions in both the single-grid and dual-grid results! Contours of surface pressure in Fig. 4 show similar behavior. From these results and the results shown next, we have concluded that this case is driven mainly by the dynamics, and the applied forcing is not enough to take control over it.

We have already mentioned that the applied forcing can create sharp gradients in the total tendency (at the forcing scale) generating noise in the solution. These small scale noise can be easily filtered out from signals relatively large in magnitude, like temperature in Fig. 3, by increasing the coefficient of artificial diffusion. However, the situation is difficult when the signal itself is of order of the generated noise; then it becomes difficult to separate the noise from the real signal. This is the situation of cloud liquid water, $Q_{w}$, in Fig. 5. Typical value of $Q_{w}$ is in the order of $10^{-4}$ which is only few order of magnitudes larger than the noise. It is for this reason, the dual-grid results show many small scale clouds as compared to the single-grid results, especially in mid-latitude. This raises a serious question on the utility of the dual-grid approach with fixed forcing scale in addressing cloud related issues.

For comparison purpose, we have also performed simulations in which the 

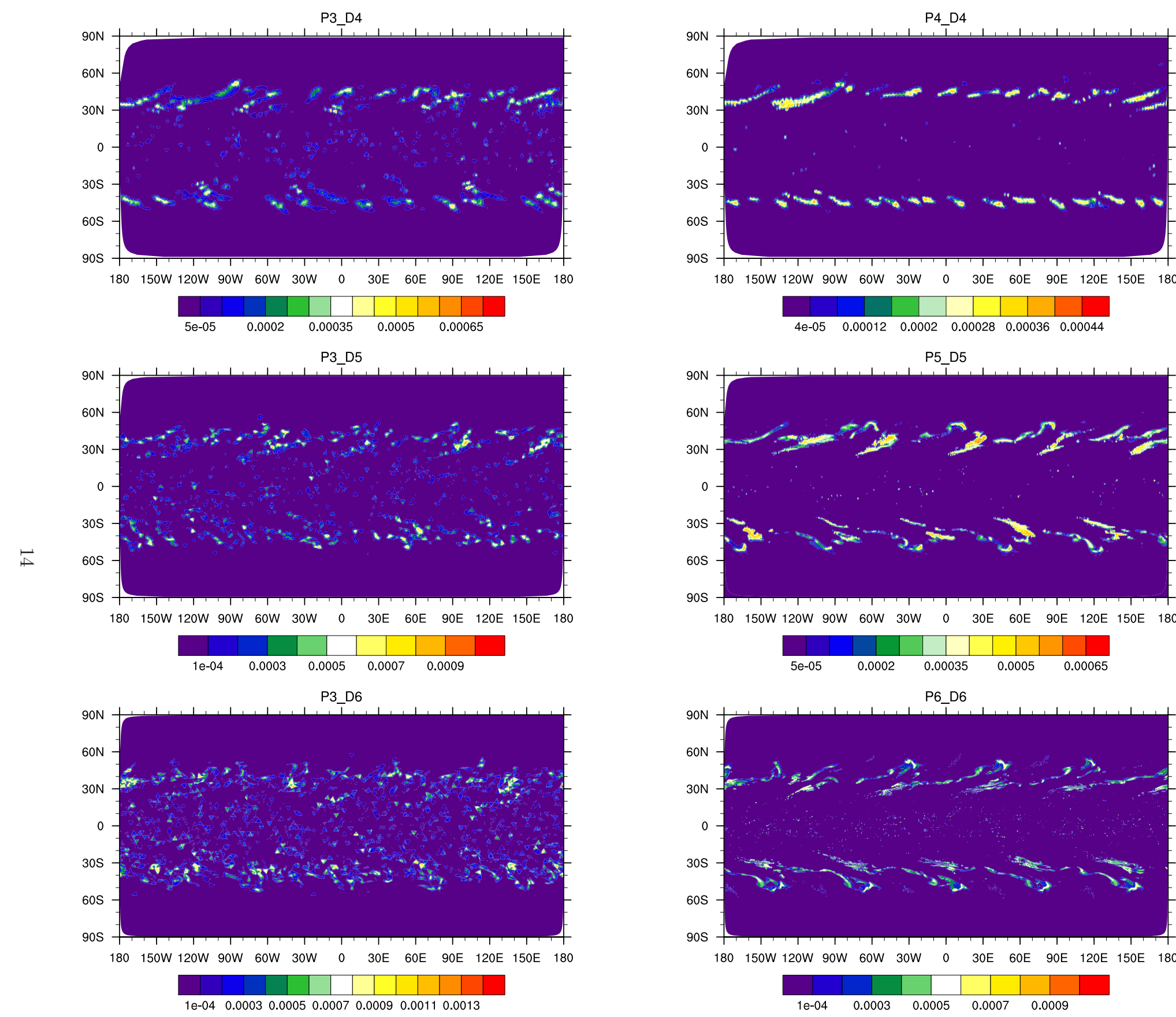

180 150W 120W 90W 60W 30W 0 SOE 60 E $90 \mathrm{E}$ 120E $150 \mathrm{E} \quad 180$

\begin{tabular}{lll|l|l|l|l|l|l|} 
& & & & & & & & \\
$4 \mathrm{e}-05$ & 0.00012 & 0.0002 & 0.00028 & 0.00036 & 0.00044
\end{tabular}

P5_D5
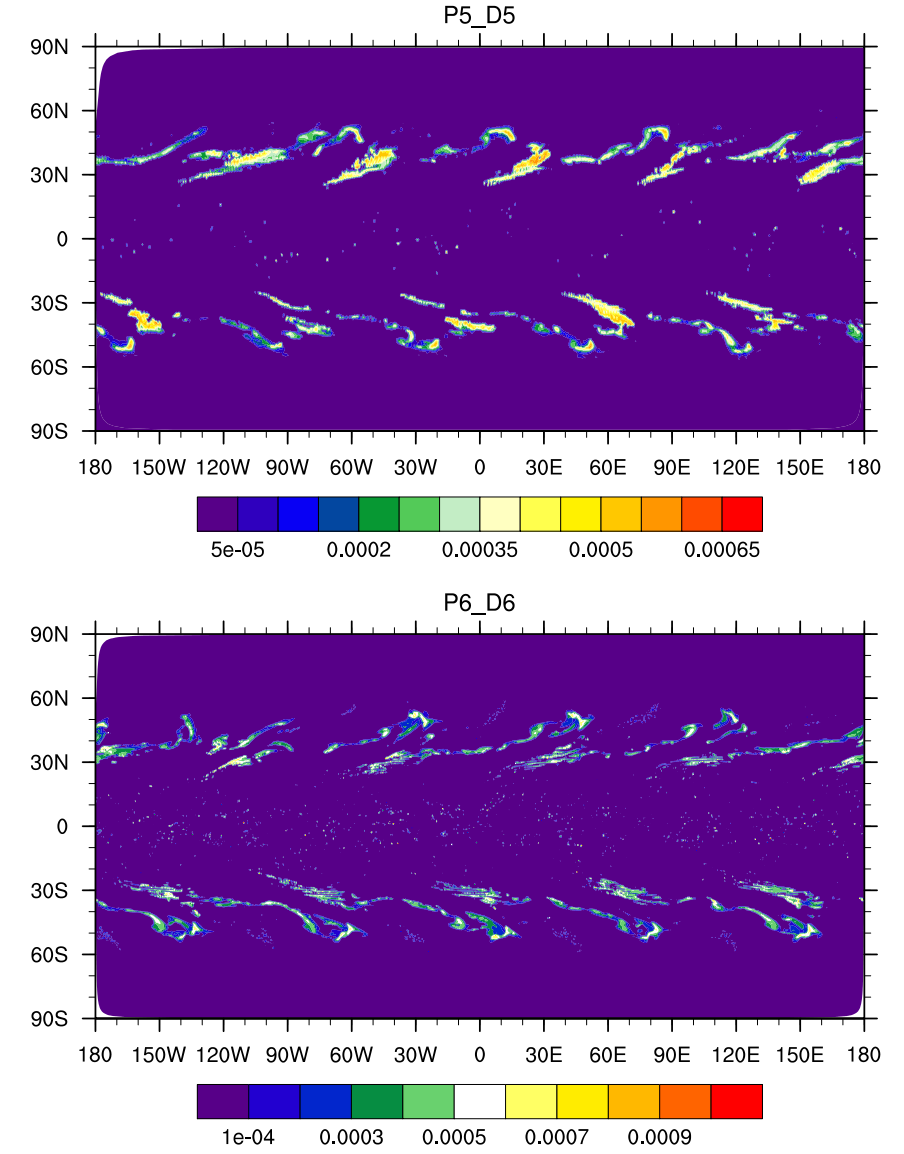

Figure 5: Cloud liquid water contours at day 10 of the simulation at an arbitrary $\eta$ level. Left-hand column shows dual-grid results and right-hand column shows the corresponding single-grid results. 
interpolation method conserved the state variables and tendencies itself. We call it a non-conservative method in the sense that it doesn't conserve global invariant quantities. Fig. 6 compares the two interpolation method for surface pressure on model day 12. Visibly, the results from both the methods appear nearly the same. One would need to integrate much longer in time to see any substantial advantage of the conservative method over the other.

The results we have shown so far are instantaneous plots at some vertical level. To understand the global convergence behavior of the dual-grid approach, we have calculated the normalized $l_{2}$ norm of selected variables at each vertical level. For a variable $\phi(\lambda, \phi, \eta)$, the normalized $l_{2}$ norm is calculated as

$$
|\phi|(\eta)=\left[\frac{\sum_{i=1}^{N} \phi_{i \eta}^{2} A_{i \eta}}{\sum_{i=1}^{N} A_{i \eta}}\right]^{1 / 2},
$$

where summation is over all the triangles $(N)$ at a given $\eta$ level, and $A_{i \eta}$ is area of $i^{t h}$ triangle at that $\eta$ level. Fig. 7 shows the $l_{2}$ norm of $Q_{w}$ and velocity divergence for the two interpolation methods used in dual-grid approach, and for the single-grid approach. All figures have been drawn on the same scale for proper comparison. For a converging solution one would expect the difference between two consecutive curves (in magnitude) to decrease consistently at all vertical levels. Which, of course, is the not the case here. In fact, the differences has increased from green to blue curve for both $Q_{w}$ and divergence fields. As before, two important things that we note in this figure are: (a) difference between the two interpolation methods is negligible, except around $\eta=25$ in $Q_{w}$ curves, and (b) velocity divergence (which should ideally be zero!) is large in dual-grid solutions due to interpolation error. 


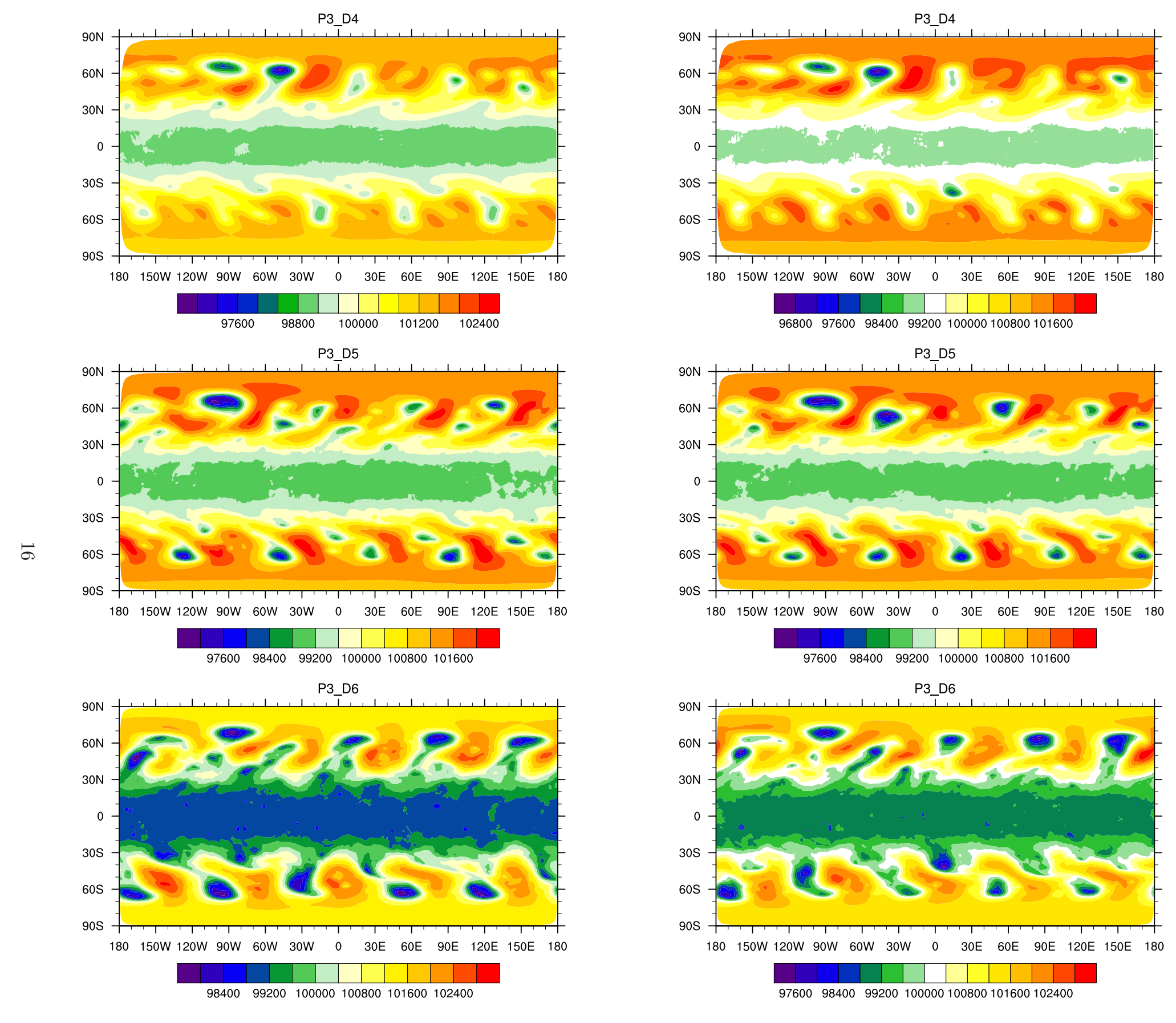

Figure 6: Contour plots of surface pressure comparing the outputs from: conservative interpolation method (left-hand column), and non-conservative method (right-hand column). 
(a)

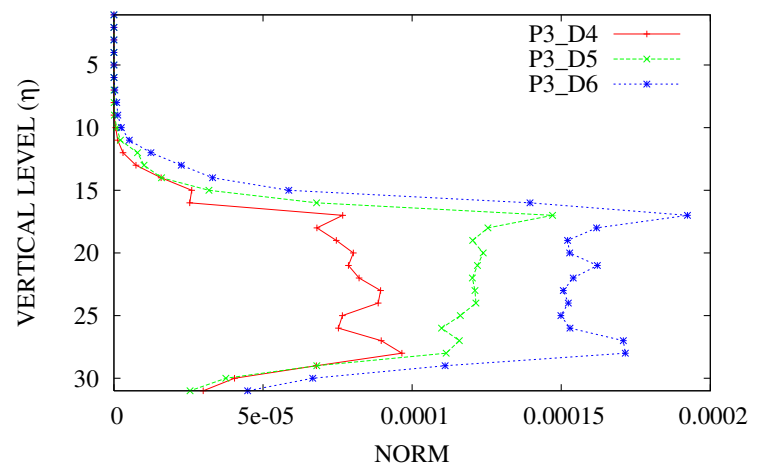

(b)

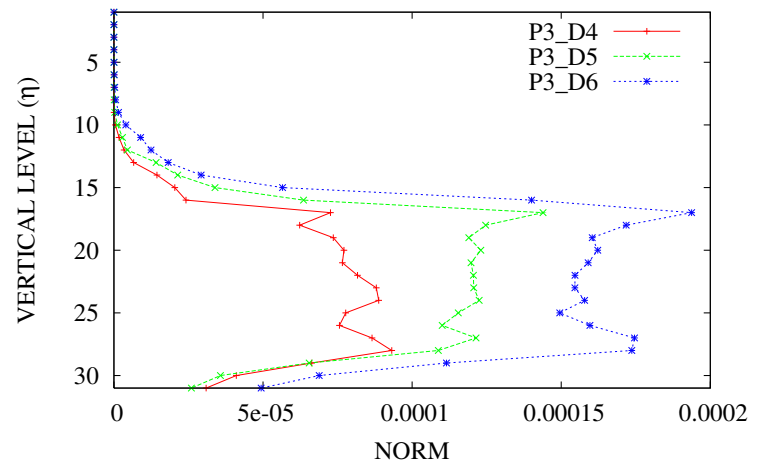

(c)

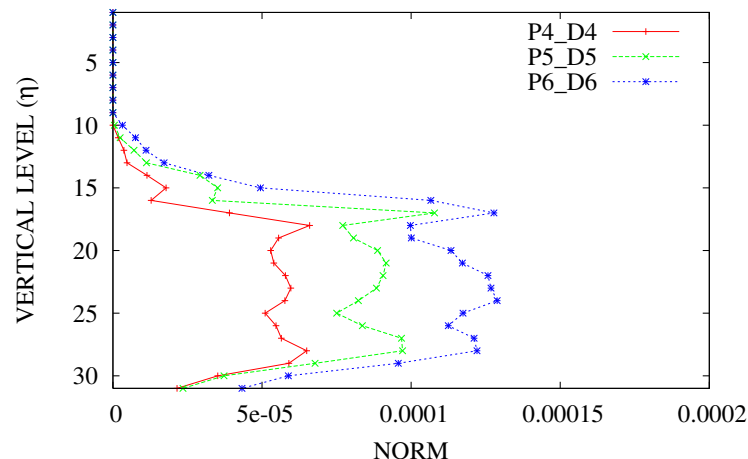

(a)

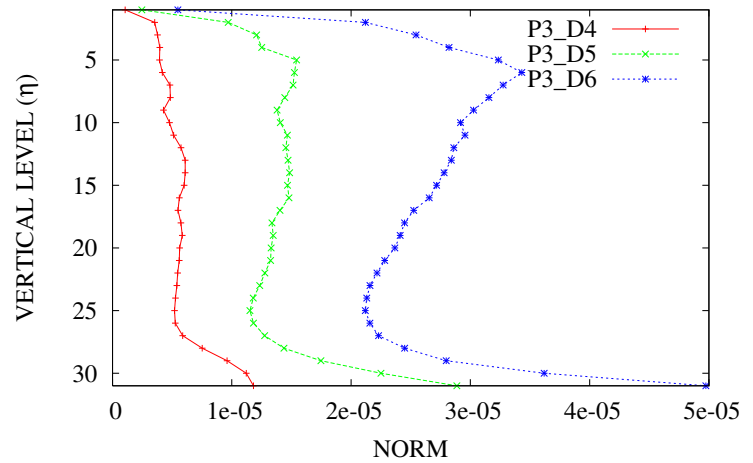

(b)

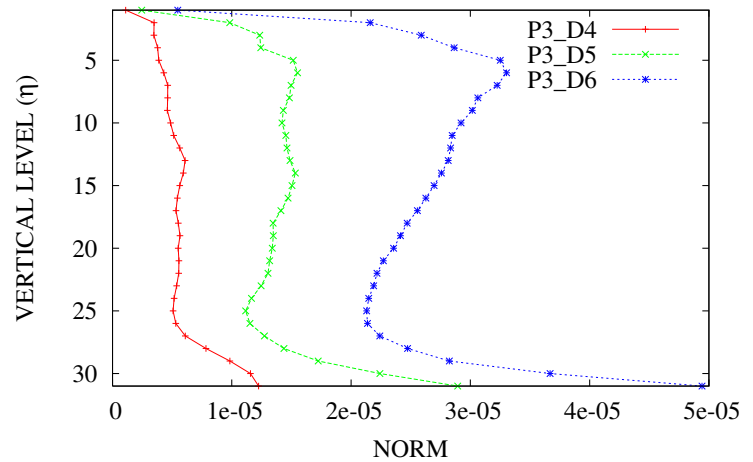

(c)

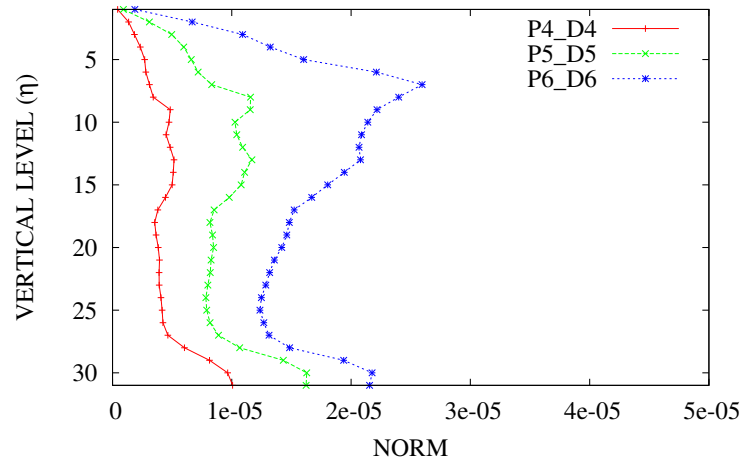

Figure 7: $l_{2}$ norm of cloud liquid water (left-hand column) and velocity divergence (right-hand column) for: (a) conservative dual-grid, (b) non-conservative dual-grid, and (c) single-grid approaches. 


\section{Part II}

\section{Development of Heterogeneous Multiscale Modeling Kernel in ICON}

\section{$5 \quad$ Heterogeneous Multiscale Modeling}

Cumulus convection has been a topic of great interest for years and is still unsolved. Various techniques, starting from the works of Manabe et al. (1965); Kuo (1965, 1974); Arakawa and Schubert (1974), have been developed (see Arakawa (2004) for recent review). One of the recent proposals was to replace the physics package entirely with a cloud resolving model (CRM) in a grid column (Grabowski, 2001; Khairoutdinov and Randall, 2001; Khairoutdinov et al., 2005). Khairoutdinov and Randall (2001) called this technique as super-parameterization (SP)- a compromise between fully empirical and global CRM. While superparameterzation can be viewed as a homogeneous multiscale model, wherein the entire physics package is replaced with a CRM, a heterogeneous multiscale model requires the capability to run a micro-scale model and classical physical parameterization simultaneously on a grid. Idea is to reduce the computational cost by replacing the classical parameterization at selective locations where small scale interactions play important role.

The CRM we have used in our model is a coarse grid version of the threedimensional (3D) University of California Los Angeles (UCLA) LES Stevens et al. (2005); Stevens (2007) in our model. A brief discussion of the UCLA-LES is given below for the sake of completeness:

- The dynamical equations are the anelastic approximation of the compressible Navier-Stokes equations.

- It uses the third order Runge-Kutta method for time integration

- The momentum advection is performed using fourth-order central difference and scalar advection uses monotone upwinding method

- The sub-grid scale turbulence model is based on classical Smagorinsky model with modifications to account for stratification effects.

- The default bulk microphysical scheme of the UCLA-LES model is the one from Seifert and Beheng (2001) and the radiation scheme is the one given in Pincus and Stevens (2009).

Some information on the ICON dynamical core is already provided in the section 3. In addition to that it is important to mention that the scalar advection in ICON is done using Semi-Lagrangian approach, and the physics package used 
in the hydrostatic mode is the same as that of MPI's ECHAM (Roeckner et al., 2003). It uses the mass flux based convection scheme of Tiedke (1989) with modifications for penetrative convection by Nordeng (1996). The scheme for the representation of stratiform clouds consists of prognostic equations for the vapor, liquid, and ice phase. It uses a cloud microphysical scheme of Lohmann and Roeckner (1996). Details of the remaining processes can be found in Roeckner et al. (2003)

The coupling mechanism between the dynamical core and the CRM providing the sub-grid scale tendency is described next following Khairoutdinov et al. (2005). The CRM (using periodicity in horizontal) integrates in time using the dynamical tendencies from GCM as large-scale (LS) forcing, and feedsback the physical tendencies as sub-grid scale tendency to the GCM. The dynamics of such a CRM is mathematically expressed for any conserved variable $\phi$ as:

$$
\frac{\partial \bar{\phi}}{\partial t}=\frac{\partial \bar{\phi}}{\partial t}_{\text {model }}+\frac{\partial \bar{\phi}}{\partial t}_{L S}
$$

where overbars denote horizontal domain averaging. The first term on the righ hand side (rhs) of Eq. 23 is the turbulent flux divergence term as calculated by the CRM:

$$
\frac{\partial \bar{\phi}}{\partial t}_{\text {model }}=-\frac{\partial \overline{w^{\prime} \phi^{\prime}}}{\partial z}
$$

where the primes denote grid box deviations from the horizontal slab average. Note that no additional terms appear in 24 when $\phi$ is a conserved variable. The second term on the rhs of Eq. 23 is the tendency from GCM, acting as LS forcing for CRM Grabowski (2001):

$$
\frac{\partial \bar{\phi}}{\partial t}_{L S}=-\overline{\boldsymbol{U}} \cdot \nabla \bar{\Phi}+D_{\Phi}
$$

Here, $\overline{\boldsymbol{U}}=(\bar{U}, \bar{V}, \bar{W})$ is the GCM component of three velocities. Note that capital letters are used for GCM variables and small letters for the CRM variables, and the overbars on GCM variables indicate grid cell averaging. The first two terms in Eq. 25 comprise the advective tendencies from GCM using the local grid values (in which the CRM sits) and terms in $D_{\Phi}$ comprise of remaining dynamical tendency terms like Coriolis term, pressure gradient term, etc. No LS forcing term has been applied to the vertical momentum equation in CRM following Grabowski (2001). Because the calculations of rain water and ice in UCLA-LES is performed in an entirely different manner than in ICON, we decided not to couple these two variables. It is hoped that as long as cloud water is properly advected in CRM (due to the applied LS forcing from GCM), the missing advective tendencies of rain water and ice will have very little impact in the lower troposphere. It is important to note that we do not couple velocities by relaxing them to each other as it was suggested in Grabowski et al. (1996). A damping layer is applied for momentum and thermodynamic equations in 
CRM's top model levels wherein the CRM computed variables are nudged to the previous time step values from GCM using a slow vertical varying function.

Feedback from CRM, acting as sub-grid scale tendency for GCM, are the turbulent flux divergence terms in Eq. 24. It is important to note that same coupling mechanism can be achieved in a simple manner by relaxing the variables to each other on the GCM time step as shown in Grabowski (2003) and used in Khairoutdinov et al. (2005). However, the experiments performed in this report do not consider the feedback from CRM. At the moment our intention is to evaluate the grid-wise behavior of classical parameterization, mainly convective parameterization, in comparison to the tendencies from CRM. In order to do so we run the ICON model with classical parameterization parallely with CRM placed in few representative columns allowing for one-way communication. That is, the GCM feeds the CRM with its dynamical tendency but the CRM doesn't feed the sub-grid scale tendencies back. So we get two response, one from classical parameterization and one from CRM, both driven by the same dynamical tendency. We believe this allows for a better understanding of the classical parameterization at much cheaper cost.

\section{Evaluating classical parameterization}

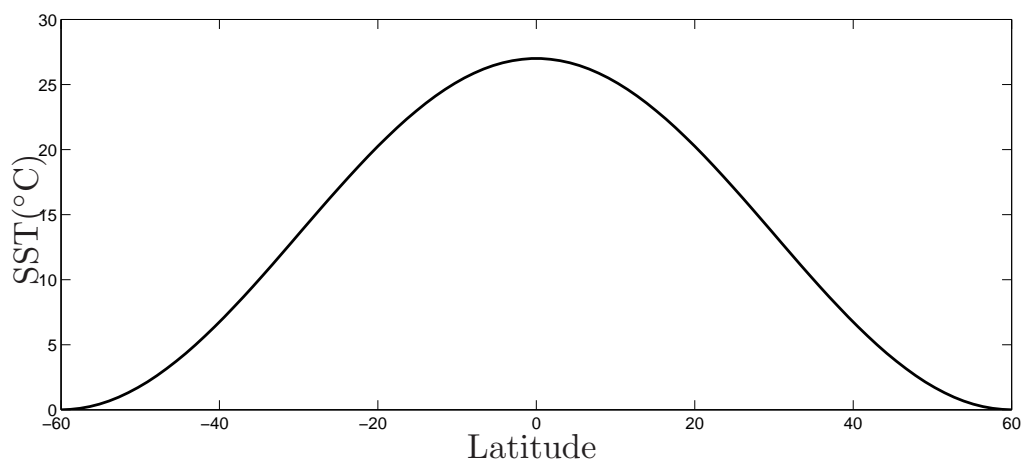

Figure 8: SST profile corresponding to Eq. 26

We have used the fixed sea surface temperature (SST) aqua-planet test suite of Neale and Hoskins (2000) for our fully prognostic multi-column experiment. The correct solutions of aqua-planet tests are not known but it is argued that these studies help us understand physical processes and their interaction with dynamical cores (Williamson and Olson, 2003). The simulated case corresponds to the CONTROL case of Neale and Hoskins (2000) in which the SST field $\left({ }^{\circ} \mathrm{C}\right)$ is given by 


$$
T_{s}(\lambda, \phi)= \begin{cases}27\left[1-\sin ^{2}(90 \phi / 60)\right], & \text { if }|\phi|<60 \\ 0, & \text { if }|\phi|>60,\end{cases}
$$

for $\lambda$ and $\phi$ being the latitude and longitude respectively. SST distribution corresponding to Eq. 26 is shown in Fig. 8. The solar forcing is fixed at March equinoctial conditions which effectively removes the seasonal variability in model runs, however, the model includes a diurnal cycle in the radiative forcing. GCM was first run in a standalone mode for 3 years starting from the initial state of Jablonowski and Williamson (2006) to ensure statistically steady state. The zonally averaged (and averaged over last 12 months) precipitation from ICON is shown in Fig. 9, overlayed with the same quantity from ECHAM, to confirm the correctness of the new GCM. ICON captures the peak precipitation organized into a single topical convergence zone same as ECHAM. Differences are visible in the secondary precipitation maxima corresponding to the mid-latitude storm tracks. We understand this difference can be fixed by tuning the parameters according to the new dynamical core. Nevertheless, the new model does produce acceptable results which has been verified by the development team at MPI.

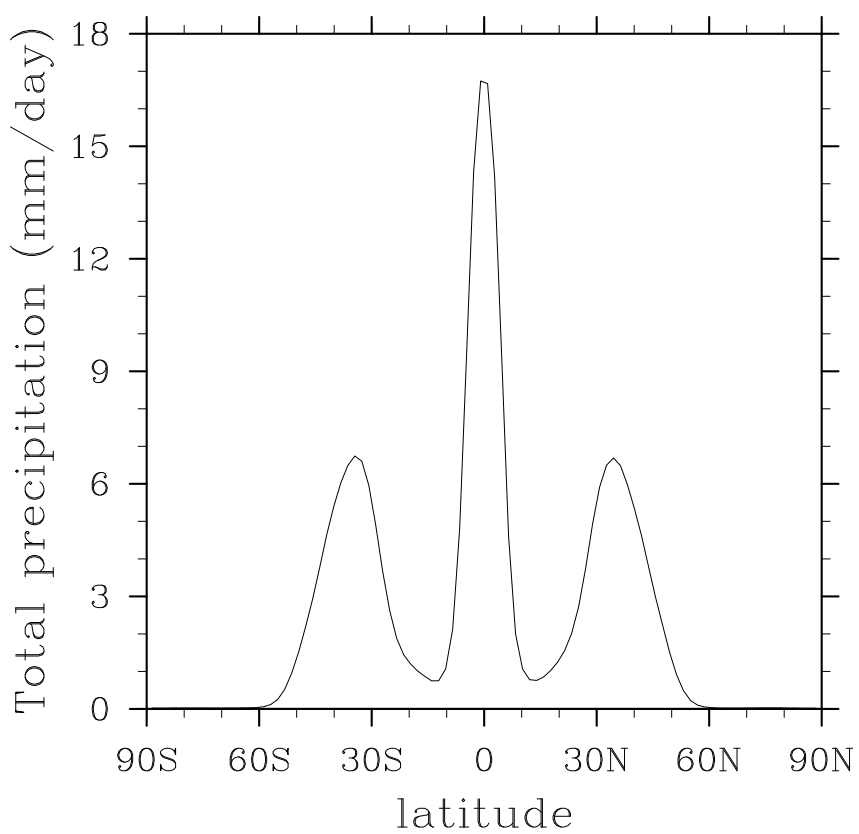

Figure 9: Zonally average, time mean precipitation rate from aqua-planet simulation using ICON

Idea of using aqua-planet simulations for evaluating cumulus convection parameterization was motivated from the findings of Medeiros and Stevens (2011), 
in which the authors demonstrated that aqua-planet suite provides an appropriate framework for evaluating tropical cloud regimes in climate models. In subsequent analysis Medeiros et al. (2008) argued that the representation of shallow cumulus convection is largely responsible for the simulated differences in the climate sensitivity of different models. Motivated from their findings we decided to first analyze the behavior of cumulus convection in shallow cloud regime.

The surface precipitation plot in Fig. 9 clearly shows that the region of lowest precipitation in the tropics is centered about $\pm 12^{\circ}$. Therefore we placed 30 equispaced CRMs along a longitudinal circle about $12^{\circ} \mathrm{N}$. Each CRM in the present study uses $16 \times 16$ points in horizontal and 30 points in vertical. All vertical levels coincide with that of GCM except for the topmost GCM level where CRM doesn't compute anything. GCM uses triangular grids whose equivalent resolution is around $100 \mathrm{~km}$ and the horizontal grid resolution in CRM is fixed at $2 \mathrm{~km}$. All the experiments use the same resolution. These CRMs are initialized with the soundings of the respective grid column at the last day of the standalone run described above. To trigger turbulence, small amplitude noise is added initially to the CRM temperature and humidity fields near the surface. CRMs then integrate in time using the LS forcing (see Eq. 25) from GCM at every GCM time step, $\Delta t_{G C M}$. As mentioned earlier, unlike usual superparameterization, CRMs in present case do not feedback. The model is run for next 6 months to get statistically meaningful results.

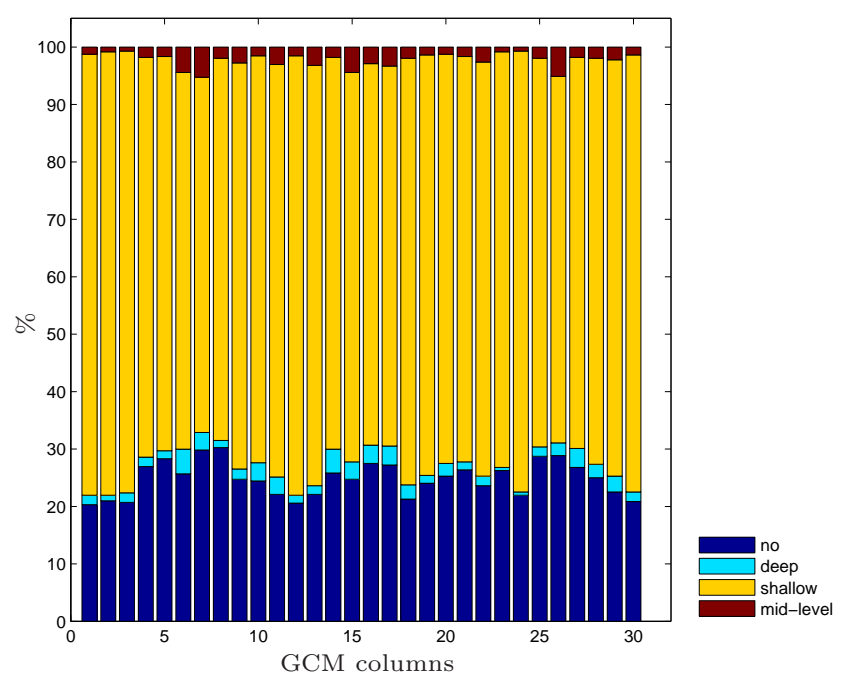

In order to show that these 30 columns actually undergo shallow cumulus parameterization, we have plotted the number of times (in percentage) a particular type of convection is triggered in each column in Fig. 6. Triggering mechanism switches on either no, shallow, deep, or mid-level convection based on local parcel buoyancy. The triggered value is stored at every GCM time step for the whole 6 month. It is clear from the figure that all 30 columns un- 
dergo shallow cumulus convection most of the time, minimum being $60 \%$. For more than $10 \%$ (and less than $35 \%$ ) of the time no convection is triggered, with occasional events of deep and mid-level convection.

\subsection{Large-scale forcing and Time-varying statistics}

The time mean LS forcing for the thermodynamic variables as experienced by the CRM is shown in Fig. 10. The last panel shows the subsidence. It is to be noted the the solid line in this figure, and the figures to be shown next, correspond to the mean of the $30 \mathrm{CRMs}$ whereas the dashed line indicate their upper and lower bound. Assuming that the participating columns are statistically independent, we define the mean and the bounds for any variable $\phi$ as

$$
\begin{array}{r}
\phi_{\text {mean }}(p)=\frac{1}{N} \sum_{i=1}^{N} \phi_{i}(p) \quad \mathrm{N}=30 \\
\phi_{\text {upperbound }}(p)=\max \left(\phi_{i}(p), \mathrm{i}=1,30\right) \\
\phi_{\text {lowerbound }}(p)=\min \left(\phi_{i}(p), \mathrm{i}=1,30\right)
\end{array}
$$

These tendencies show the typical behavior in shallow cumulus regime where the subsidence causes large-scale warming and drying. We begin by examining the time evolution of daily mean of cloud related quantities. All time evolution plots show the mean (solid line), the lower and upper bounds (dashed line) of the ensemble of 30 columns. These quantities are defined in the same manner as in Eq. 27 by replacing $p$ with $t$. It is to be noted that for some of the figures where mean and bounds are largely separated no bounds are shown for the purpose of clarity.

Time evolution of vertically integrated liquid water path (LWP) and surface precipitation is shown in Fig. 11. It is clear to note the two are quite in phase and that GCM shows more undershoots than CRM suggesting slightly lesser LWP and precipitation in GCM. Sensible heat flux in Fig. 12(a) show consistently lower value of heat flux in CRM which suggests that the temperature difference between the surface and the first model level in CRM is lower in CRM than in GCM. Because the SST is fixed in both GCM and CRM, and the heat flux is positive, we can conclude that the first model level temperature in CRM is slightly higher than in GCM. This can be seen in the vertical profile of liquid water potential temperature $\left(\theta_{l}\right)$ in Fig. 13(a). The latent heat flux in Fig. 12(b), however, show just opposite. It is consistently higher in CRM indicating higher value of specific humidity $\left(q_{v}\right)$ in the first model level of CRM as compared to GCM.

We then show the time evolution of top of atmosphere fluxes of the two models in Fig. 12(c) and (d). Expectedly, the two two quite similar trend with some differences. Short-wave incoming flux is slightly higher in CRM than in GCM the reason for which is not known. It could be because of the different radiation scheme used in CRM which randomly samples the spectra in heating rate calculation. Hopefully, the two produce same influx when run 

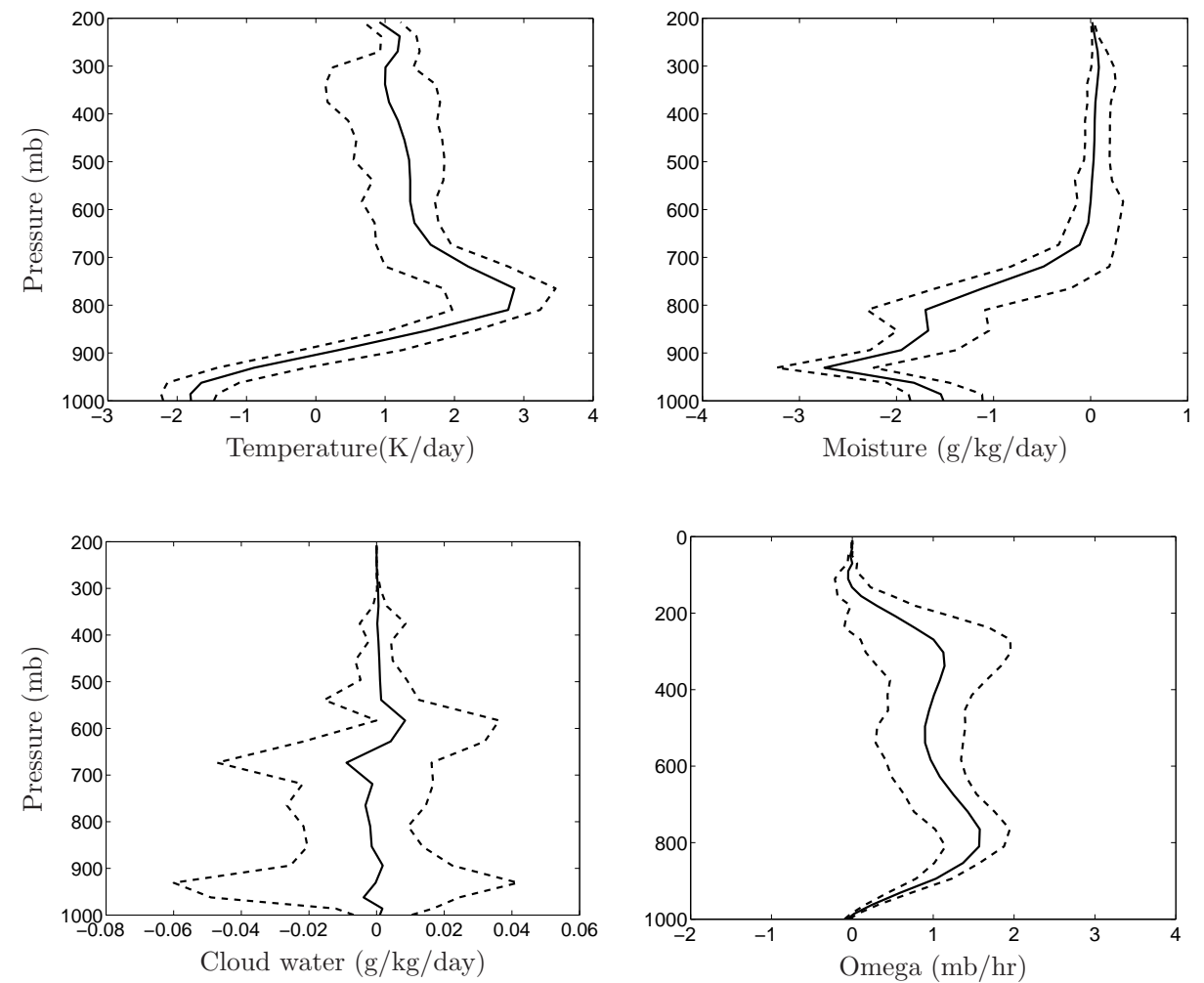

Figure 10: Time mean of LS forcing fed to CRMs in control case. The solid lines indicate the mean and dashed lines indicate the upper/lower bounds of the column ensemble

(a)

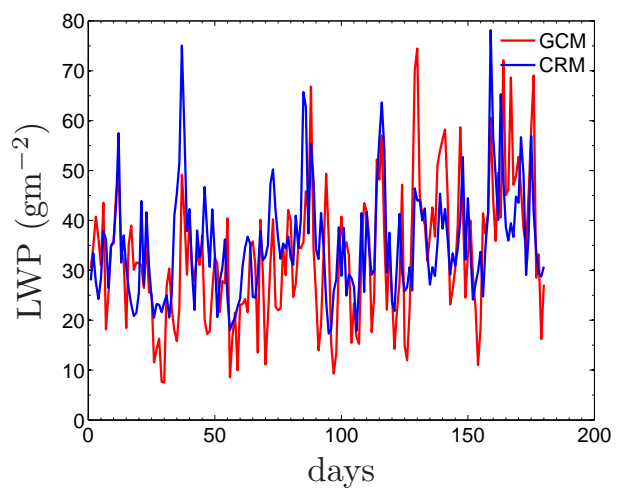

(b)

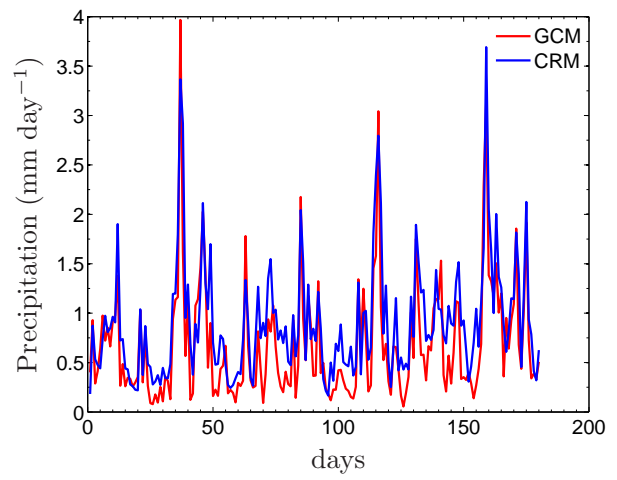

Figure 11: Time evolution of daily mean liquid water path (LWP) and surface precipitation rate for GCM columns and CRMs. Only mean of the 30 columns is shown for the purpose of clarity. 
for sufficiently longer time. The differences in the bounds in long-wave TOA flux indicate that columns wise GCM and CRM differ significantly but not in the mean sense. We suspect the statistical nature of the radiation algorithm for this behavior.

(a)

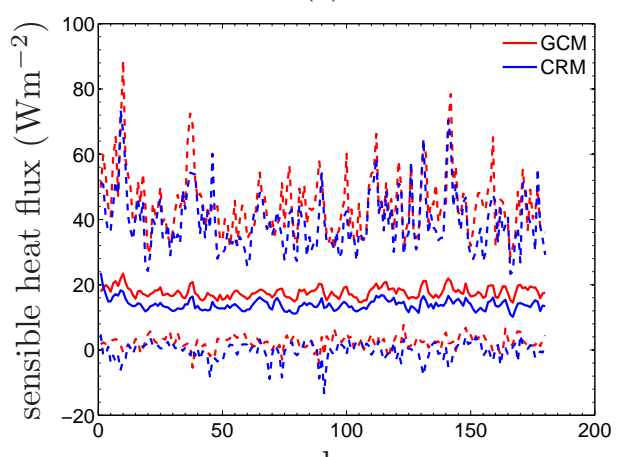

(c) days

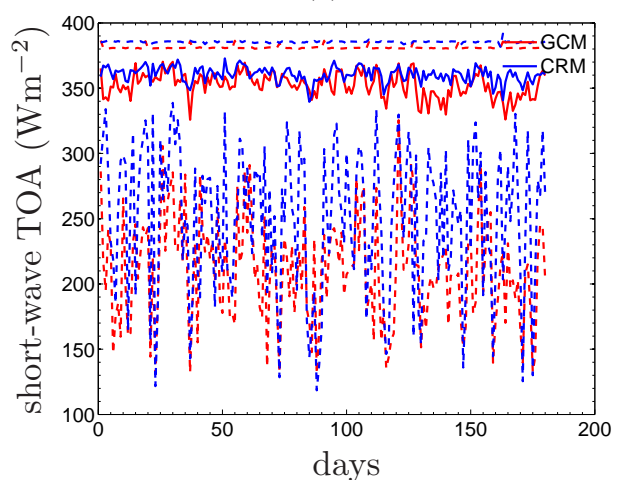

(b)

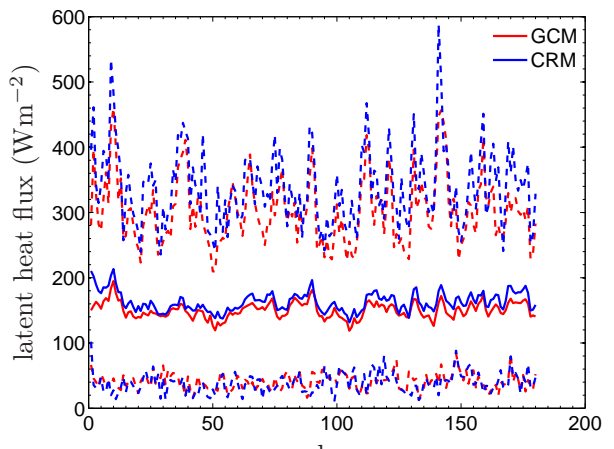

(d)

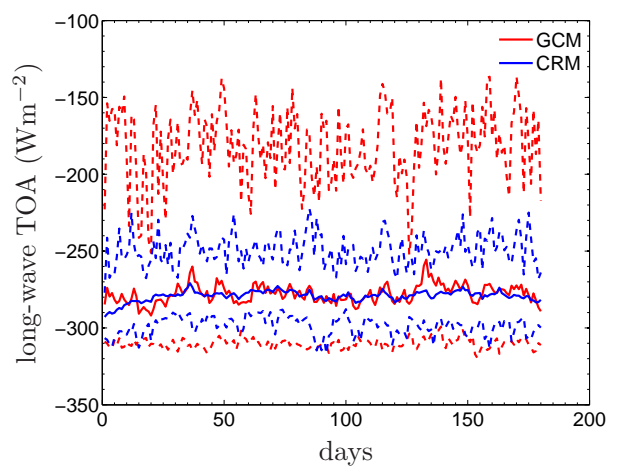

Figure 12: Time evolution of daily mean (a) sensible heat flux, (b) latent heat flux, (c) short-wave TOA flux, and (d) long-wave TOA flux for GCM and CRM columns. The solid lines indicate the mean and dashed lines indicate the upper/lower bounds of the column ensemble.

Liquid water path and surface precipitation are derived quantities values of which are bound to differ in two models using different set of equations. It will be more meaningful to compare basic thermodynamic variables like liquid water potential temperature $\left(\theta_{l}\right)$, moisture $\left(q_{v}\right)$, and cloud water $\left(q_{w}\right)$. In figures below we show both time evolution and time mean plot of these quantities. Time evolution plots show contours of daily mean averaged over 30 columns, and the time mean plot shows the vertical profile of the mean of the contours along with the upper and lower bounds of the column ensemble. Very small differences exist between GCM and CRM results beyond $p=200 \mathrm{hPa}$ because of the treatment of sponge layer described in Sec. 5. Therefore, we only show the part $p>200 \mathrm{hPa}$ in the following figures. 


\subsection{Liquid water potential temperature budget}

In order to understand the differences between GCM columns and CRMs, we will consider their budget separately. Budget equation of liquid water potential temperature $\left(\theta_{l}\right)$ for GCM columns can be written as

$$
\frac{\partial \bar{\Theta}_{l}}{\partial t}=\frac{\partial \bar{\Theta}_{l}}{\partial t}{ }_{\text {model }}+\frac{\partial \bar{\Theta}_{l}}{\partial t}{ }_{L S},
$$

where the first term on the right-hand side (RHS) is comprised of

$$
{\frac{\partial \bar{\Theta}_{l}}{\partial t}}_{\text {model }}={\frac{\partial \bar{\Theta}_{l}}{\partial t}}_{c c}+{\frac{\partial \bar{\Theta}_{l}}{\partial t}}_{v d i f}+{\frac{\partial \bar{\Theta}_{l}}{\partial t}}_{r a d},
$$

where, $c c$ stands for contribution from "convection" and stratiform "cloud" parameterization, vdif stands for contribution due to vertical diffusion, and rad is radiation term. Note that ICON solves for absolute temperature, and the effects of convection and phase change related latent heat (in stratiform clouds scheme) are paramterized, whereas CRM resolves the convection and the phase change effects are prognosed. We address the tendencies due to phase change as "cloud" related tendencies, and for simplicity we have clubbed these two terms together. The last term on RHS of Eq. 28 is the GCM dynamical tendency term as given in Eq. 25. Because this term is same for both GCM and CRM, we only discuss the "model" tendency term in figures below. Budget equation for CRM can be obtained by replacing $\Theta_{l}$ in above equations with $\theta_{l}$.

(b)
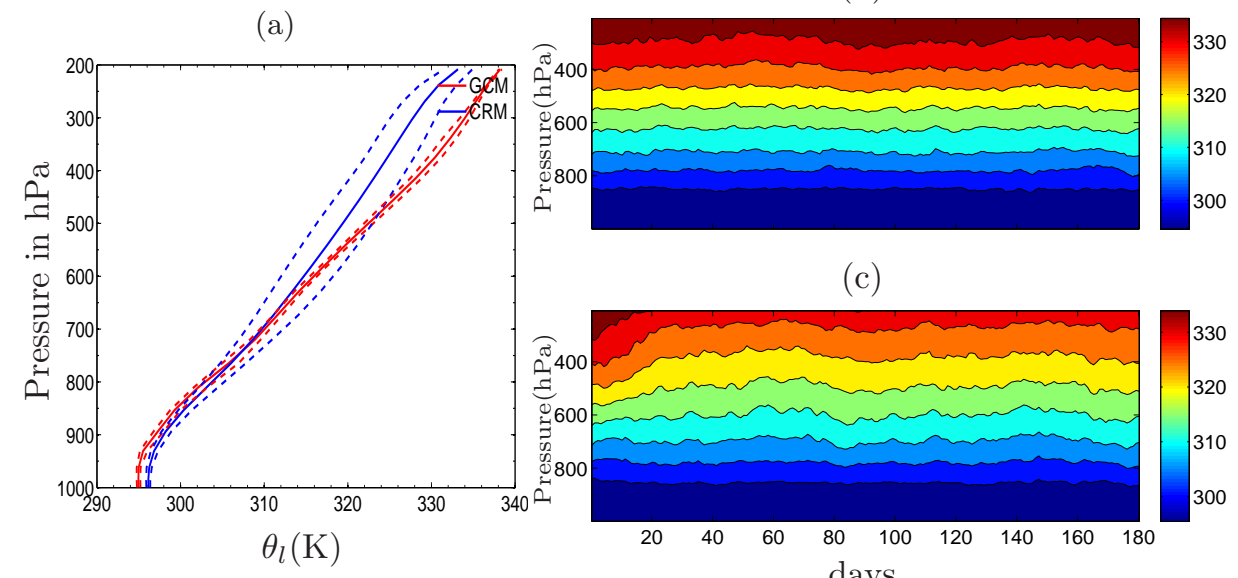

(c)

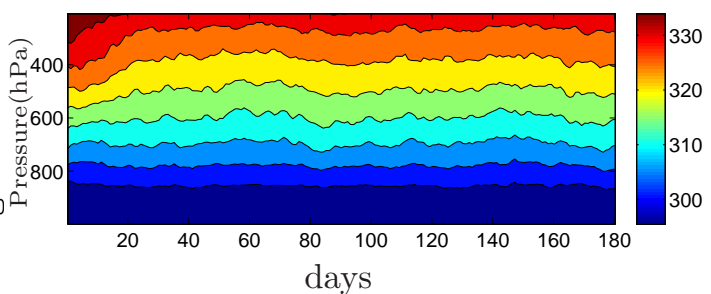

Figure 13: (a) Time mean of $\theta_{l}$. The solid lines indicate the mean and the dashed lines are the upper and lower bounds of the column ensemble; contours of column mean daily averaged $\theta_{l}$ (b) GCM columns and (c) CRMs.

Contours of $\theta_{l}$ in Fig.13(b) and (c) show that CRM (Fig. 13(b)) undergoes some variability in time in the mid troposphere $(\mathrm{p} \approx 700 \mathrm{hPa})$ which is nearly absent in GCM. The differences are larger in the upper troposphere $(\mathrm{p}<500 \mathrm{hPa})$ 
where CRM has lower temperature $\left(\theta_{l}\right)$ than GCM. This is also seen in the temporal mean plot in Fig. 13(a), indicating the presence of a source or a sink of heat in CRM. This will be explored while discussing the budget of $\theta_{l}$ in next figure. Figure 13(a) also show that the spread in CRM ensemble is quite significant compared to the spread in GCM column ensemble. This is expected because CRM solves a set of prognostic equations for its subgrid-scale physics whereas GCM parameterization is purely diagnostic and deterministic (Jones and Randall, 2011).

The temperature budget of both GCM and CRM ensemble is shown in Fig. 14. During our investigation we noted that (results not shown) convection has warming effect, for all ensemble members of both GCM and CRM, whereas the cloud related tendencies, mostly, tries to counter it. The net effect of these counteracting tendencies is heating in both CRM and GCM, except near surface and $\mathrm{p}=750 \mathrm{hPa}$ (see Fig. 14(a)). The effect of vertical diffusion is heating near the surface opposing the cooling effect of convection and cloud related tendencies as seen Fig. 14(b). It is the radiation effect that finally dominates the differences between the two by generating higher cooling rates in CRM than in GCM as seen in Fig. 14(c). This explains the seen difference in their temperature profile in Fig. 13(a). The combined effect of all these component tendencies is shown in Fig. 14(d) as $\left(\frac{\partial \bar{\theta}_{l}}{\partial t}-\frac{\partial \bar{\Theta}_{l}}{\partial t}\right)_{\text {model }}$, and in Fig. 14(e) as time evolution of column mean model tendency $\left(\frac{\partial \bar{\theta}_{l}}{\partial t}\right.$ model $)$.

\subsection{Moisture budget}

Like $\theta_{l}$, budget equation for moisture mixing ratio $q_{v}$ can also be expressed in terms of tendencies due to $c c$, vdif, and $L S$, without the radiation term. Note that the vertical diffusion term in CRM is actually computed for the total liquid water but we assume in following that the contribution of cloud water in vertical diffusion is negligible.

Moisture profile in Fig. 15(a) indicate large mixing ratio in CRM throughout the troposphere. The slightly larger value of latent heat flux in CRM in Fig. 12 (b) is due to the high value of $q_{v}$ in first model level in CRM. The time evolution plot in Fig. 15(c) show early development of moisture near surface before it reaches a near steady value by day 3 . Unsteadiness is seen in both GCM and CRM near $p=700 \mathrm{hPa}$, where the difference between the two is maximum (see Fig. 15(a)). In order to understand these differences, we show component tendencies of moisture budget in Fig. 16. Moisture supplied at the lower levels through surface evaporation is transported upward predominantly by diffusion till $p=900 \mathrm{hPa}$ in both GCM and CRM. Above this level, $c c$ (cloud and convection) takes over to transport it further up in the atmosphere. It is important to realize that the differences between CRM and GCM tendencies are generally small except for $\mathrm{p}>850 \mathrm{hPa}$ (note that $\mathrm{p}=850 \mathrm{hPa}$ is cloud top, to be seen next). Although the differences are larger in this region $(\mathrm{p}>850 \mathrm{hPa})$, the $c c$ and $v$ dif terms counter each other so that the net effect is very less (see Fig. 16(c)). The combined effect of all these component tendencies is shown in Fig. 
(a)

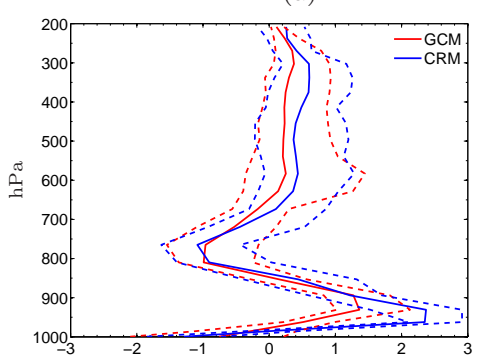

(c)

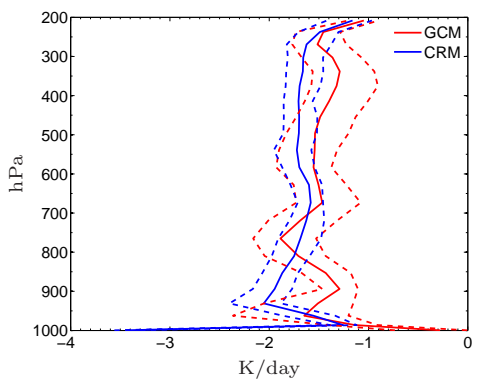

(b)

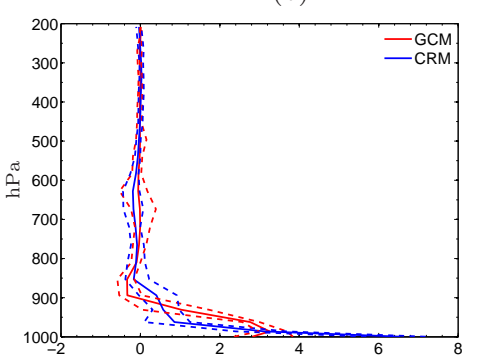

(d)

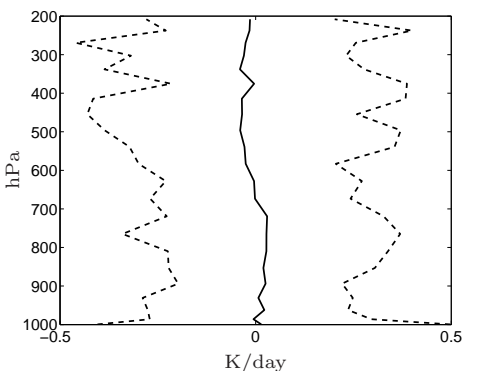

(e)

GCM

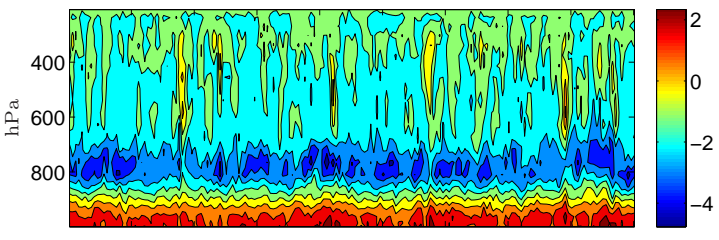

CRM

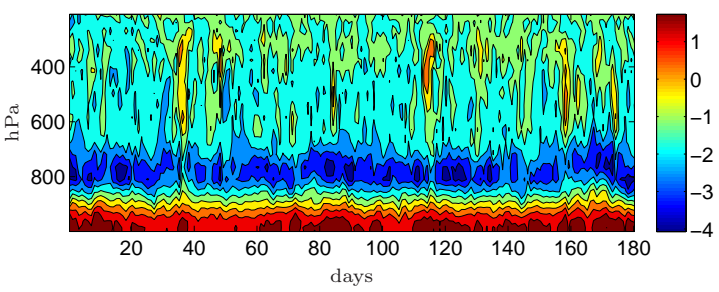

Figure 14: Time mean of different components of model tendencies in Eq. 29: (a) convection and cloud, (b) vertical diffusion, (c) radiation, (d) difference between CRM and GCM model tendencies $\left(\frac{\partial \bar{\theta}_{l}}{\partial t}-\frac{\partial \bar{\Theta}_{l}}{\partial t}\right)_{\text {model }}$, and (e) contours of four hourly averaged column mean of model tendencies of GCM and CRM. Solid lines indicate the mean and dashed lines are the upper and the lower bounds. 
(b)
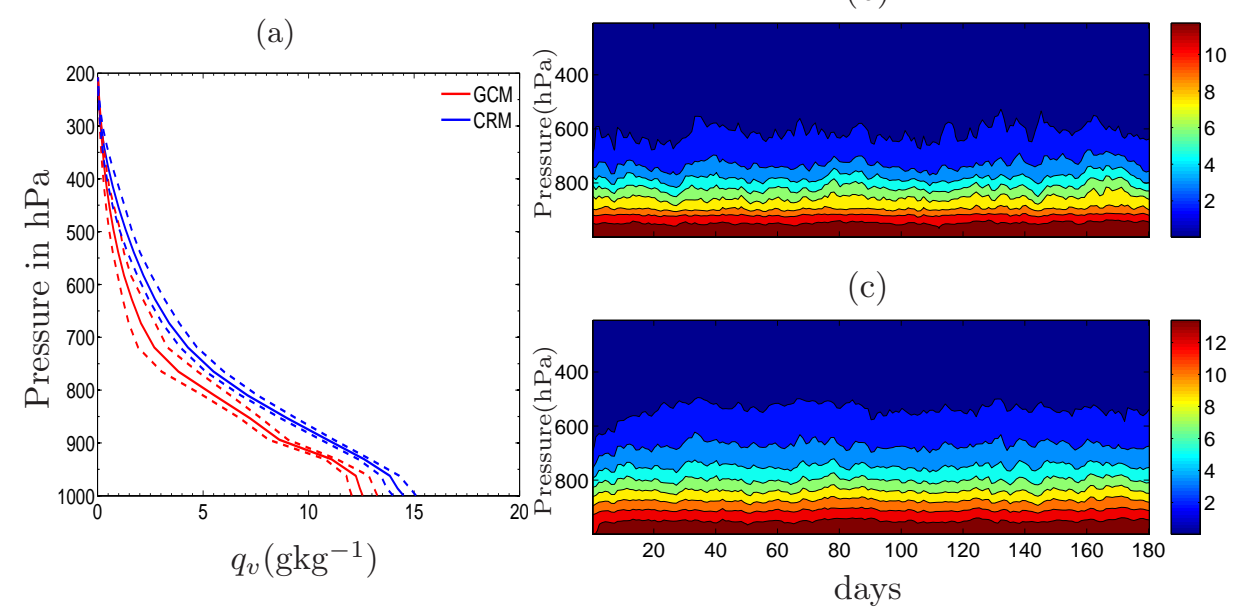

Figure 15: Same as Fig. 13 for moisture $q_{v}$ in $\mathrm{gkg}^{-1}$.

16(c) as $\left(\frac{\partial \overline{q_{v}}}{\partial t}-\frac{\partial \overline{Q_{v}}}{\partial t}\right)_{\text {model }}$, and in Fig. 16(d) as time evolution of column mean model tendency $\left(\frac{\partial \bar{q}_{v}}{\partial t}\right.$ model $)$. From Fig. 16(c) we note that net effect is relative moistening of majority of CRM columns, which explains the differences between them seen in Fig. 15(a). The peak near $\mathrm{p}=600 \mathrm{hPa}$ is because of the difference in $c c$ term in CRM and GCM. This peak is also visible in Fig. 16(d) which corresponds to the unsteadiness noted earlier in Fig. 15(b)-(c). Overall, the net tendencies from the two match qualitatively, and the differences are mainly due to the diffusion term in the boundary layer and between $850>p>650 \mathrm{hPa}$, and the $c c$ term in remaining troposphere. It is to be noted that (results now shown) the $c c$ term is dominated by negative convective tendency till $\mathrm{p}=900 \mathrm{hPa}$, and above that both convection and phase change related tendencies are positive but the latter dominates.

\subsection{Cloud water budget}

With the assumption that the diffusion term is negligible in cloud water budget equation, that we checked with the cloud water diffusion term in GCM, the only term left is due to $c c$. In ICON, the convective scheme passes the detrained liquid water to the cloud scheme which then calculates the parameterized tendency for large-scale. Therefore, the $c c$ term in ICON only contains tendencies due to phase changes. Since it is difficult to make such classification in CRM, we assume that the cloud water convective tendency in CRM is negligible and the $c c$ term only contains phase change related tendencies.

As we noted in the LWP plot, the GCM has slightly higher content of cloud liquid water (see Fig. 17(a)), although the more interesting feature is vertical profile of $q_{w}$ and its behavior in time in GCM. The vertical profile of $q_{w}$ in Fig. 17(a) show three peaks in GCM at $p=950,750$ and $680 \mathrm{hPa}$ which is not visible in CRM. CRM vertical profile is very continuous with a single peak 
(a)

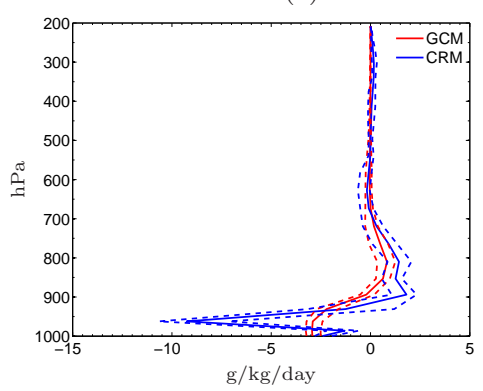

(c)

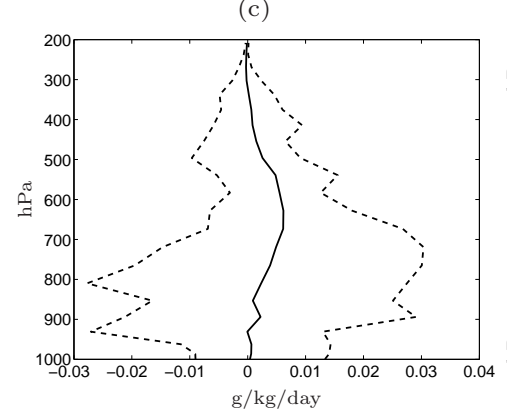

(b)

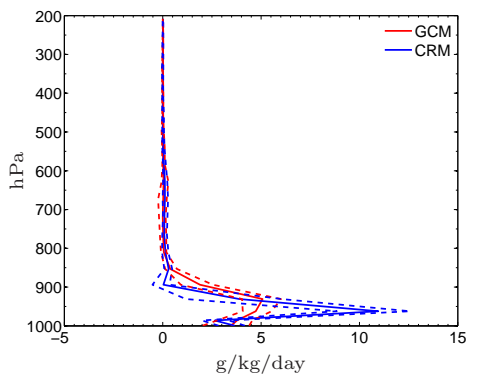

(d)

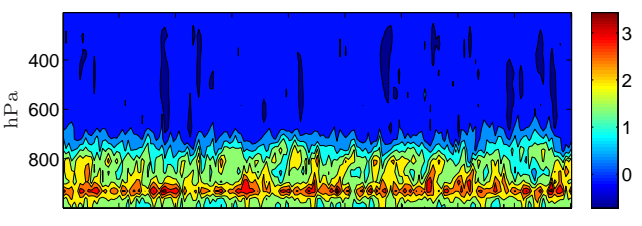

CRM

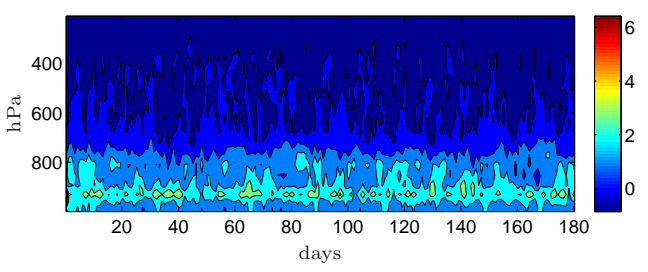

Figure 16: Time mean of different components of moisture model tendency: (a) convection and cloud, (b) vertical diffusion, (c) difference between CRM and GCM model tendencies $\left(\frac{\partial \overline{q_{v}}}{\partial t}-\frac{\partial \overline{Q_{v}}}{\partial t}\right)_{\text {model }}$, and (d) contours of four hourly averaged column mean of model tendencies of GCM and CRM. Solid lines indicate the mean and dashed lines are the upper/lower bounds.

(b)
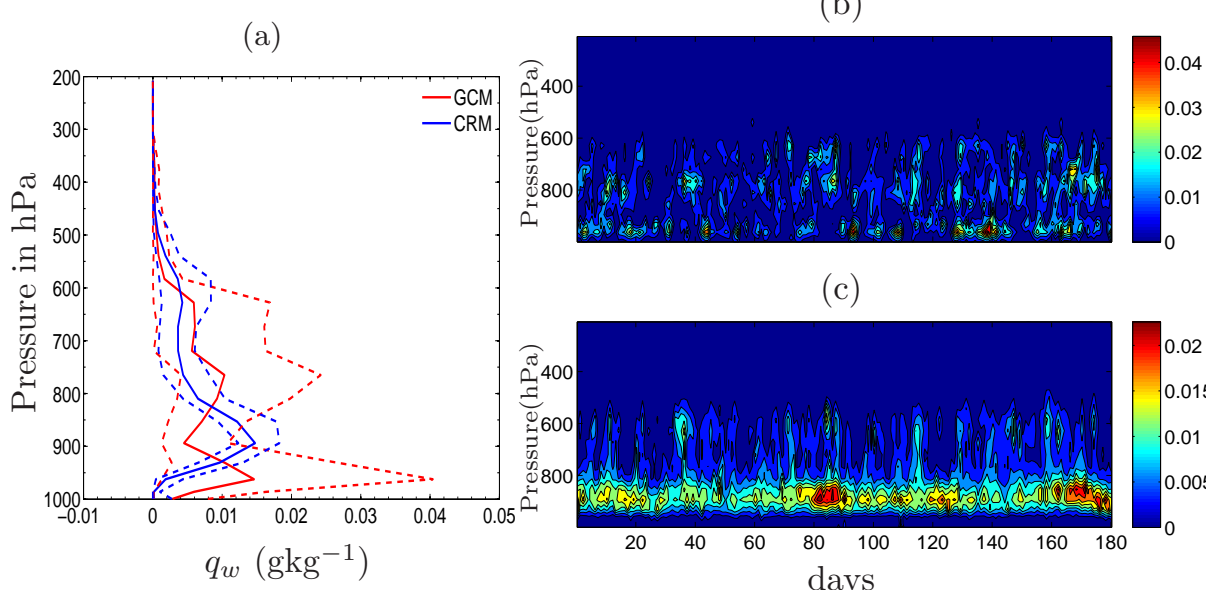

(c)

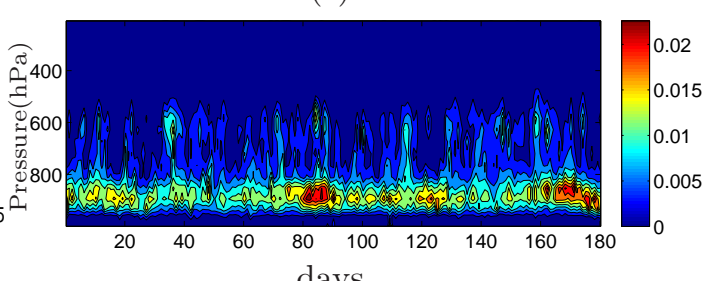

days

Figure 17: Same as Fig. 13 for moisture $q_{w}$ in $\mathrm{gkg}^{-1}$. 
(a)

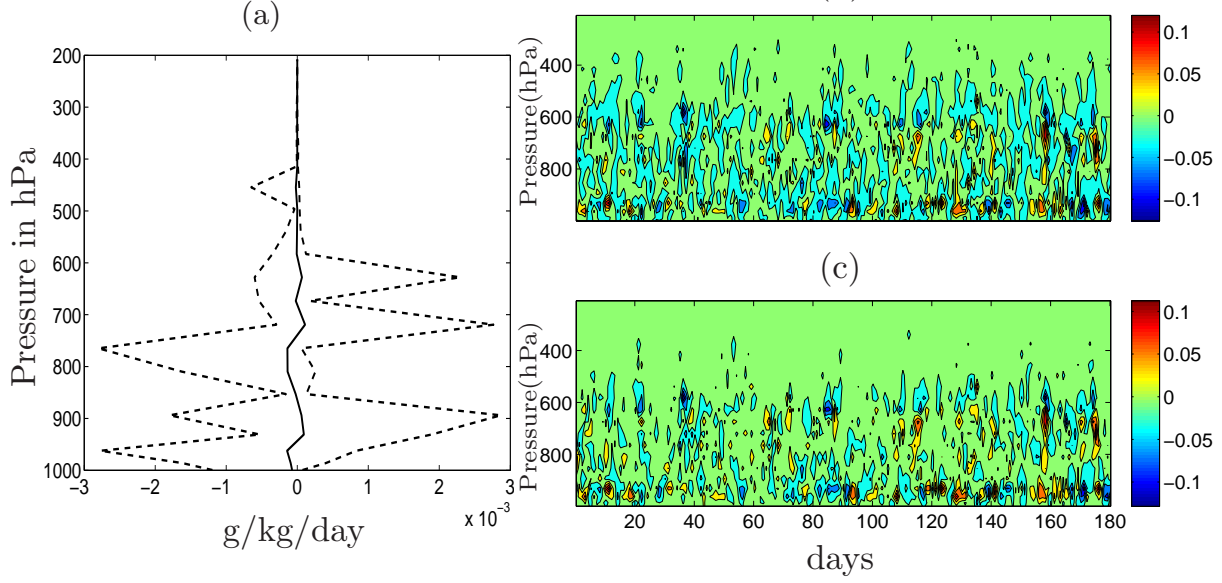

Figure 18: (a) Time mean of difference between CRM and GCM model tendencies $\left(\frac{\partial \overline{q_{w}}}{\partial t}-\frac{\partial \overline{Q_{w}}}{\partial t}\right)_{\text {model }}$, and contours of four hourly averaged column mean of model tendencies of GCM (b) and CRM (c). Solid lines indicate the mean and dashed lines are the upper/lower bounds.

at $p=900 \mathrm{hPa}$. Also, the first peak in GCM appears to be very close to the surface, in addition, the near surface $q_{w}$ in GCM is non-zero. Spread within GCM columns are also very large as compared to CRM. Similar behavior is also noted in Fig. 17 (b) and (c) where GCM shows discontinuity in time as opposed to CRM. Such discontinuous behavior in GCM is sign of the empiricism associated with the physics modeling.

While the difference between cloud water profiles in GCM columns and CRMs is quite significant, the difference between their tendencies is not. However, it is to be noted that the even a small difference in tendency can cause large accumulative effect, in addition to the differences in their vertical distribution. The total tendency difference between the two can be seen in Fig. 18. Some of the CRMs have positive excess cloud water tendency over GCM while some have negative, so that the mean stays very close to zero but on the negative side. The contours in Fig. 18(b) show many spots of marginally strong tendencies, near $p=900 \mathrm{hPa}$ and $p=600 \mathrm{hPa}$, in CRM that are totally absent in GCM. GCM, however, does capture spots of strong tendencies. 


\section{Summary}

We summarize the report by listing the things we have achieved so far and the things we intend to do in the near future.

\section{Achievements:}

- Implemented a fully parallel version of dual-grid approach in main frame code ICON.

- Implemented a fully conservative first-order interpolation scheme for horizontal remapping.

- Integration of UCLA-LES micro-scale model into ICON to run parallely in selected columns. Option to the micro-scale model in parallel with the classical parameterization.

- Option to run the micro-scale model on a "physics grid" independent of the dynamics grid.

- Cloud feedback studies on aqua-planet setup to evaluate the classical parameterization on a small domain.

\section{Things to do:}

- Run the existing model with aqua-planet setup on global scale.

- Study the low cloud feedback in a perturbed climate 


\section{References}

Arakawa, A., 2004: The cumulus parameterization problem: Past, present, and future. J. Climate, 17, 2493-2525.

Arakawa, A. and W. H. Schubert, 1974: Interaction of a cumulus cloud ensemble with the large-scale enviornment, part i. J. Atmos. Sci., 31, 674-701.

Bonaventura, L. and T. Ringler, 2005: Analysis of discrete shallow-water models on geodesic Delaunay grids with C-type staggering. Mon. Wea. Rev., 133, $2351-2373$.

Dukowicz, J. and J. W. Kodis, 1987: Accurate conservative remapping for arbitrary Lagrangian-Eulerian computations. SIAM J. Sci. Stat. Comput., 8, 305-321.

Grabowski, W., 2001: Coupling cloud processes with the large-scale dynamics using the cloud-resolving convection parameterization (crcp). J. Atmos. Sci., 58, 978-997.

Grabowski, W., 2003: An improved framework for superparameterization. J. Atmos. Sci., 61, 1940-1952.

Grabowski, W., X. Xu, and M. W. Moncrieff, 1996: Cloud-resolving modeling of topical cloud systems during phase iii of gate. part i: Two-dimensional experiments. J. Atmos. Sci., 53, 3684-3709.

Jablonowski, C. and D. L. Williamson, 2006: A baroclinic instability test case for atmospheric model dynamical cores. Q. J. R. Meteorol. Soc., 132, 29432976 .

Jones, P. W., 1999: First- and second-order conservative remapping schemes for grids in spherical coordinates. Mon. Wea. Rev., 127, 2204-2210.

Jones, T. R. and D. Randall, 2011: Quantifying the limits of convective parameterizations. J. Geophys. Res., 116 (D08210).

Khairoutdinov, M. and D. Randall, 2001: A cloud resolving model as a cloud parameterization in the ncar community climate system model: Preliminary results. Geophys. Res. Lett., 28, 3617-3620.

Khairoutdinov, M., D. Randall, and C. Demott, 2005: Simulations of the atmospheric general circulation using a cloud-resolving model as a superparameterization of physical processes. J.Atmos. Sci., 62, 2136-2154.

Kuo, H. L., 1965: On formation and intensification of tropical cyclones through latent heat release by cumulus convection. J .Atmos. Sci., 22, 40-63.

Kuo, H. L., 1974: Further studies of the parameterization of the influence of cumulus convection on large-scale flow. J .Atmos. Sci., 31, 1232-1240. 
Lander, J. and B. Hoskins, 1997: Believable scales and parameterizations in spectral transform model. Mon. Wea. Rev., 125, 292-303.

Lauritzen, P. and R. Nair, 2008: Monotone and conservative Cascade Remapping between Spherical grids (CaRS): regular latitude-longitude and cubedsphere grids. Mon. Wea. Rev., 136, 1416-1432.

Lohmann, U. and E. Roeckner, 1996: Design and performance of a new cloud microphysics scheme developed for the ECHAM4 general circulation model. Clim. Dyn., 12, 557-572.

Manabe, S., J. Smagorinsky, and R. F. Strickler, 1965: Simulated climatology of a general circulation model with a hydrologic cycle. Mon. Wea. Rev., 93, 769-798.

Medeiros, B. and B. Stevens, 2011: Revealing differences in gcm representations of low clouds. Clim. Dyn., 36, 385-399.

Medeiros, B. et al., 2008: Aquaplanets, climate sensitivity, and low clouds. J. Climate, 21, 4974-4991.

Molod, A., 2009: Running GCP physics and dynamics on different grids: algorithm and tests. Tellus, 61A, 381-393.

Neale, R. B. and B. J. Hoskins, 2000: A standard test for agcms including their physical parameterizations: I: The proposal. Atmos. Sci. Lett., 1, 101-107.

Nordeng, T. E., 1996: Extended versions of the convective parameterization scheme at ecmwf and their impact on the mean and transient activity of the model in the tropics. Tech. Rep. 206, ECMWF, Reading, UK.

Pincus, R. and B. Stevens, 2009: Monte carlo spectral integration: a consistent approximation for radiative transfer in large eddy simulations. J. Adv. Model. Earth Sys., 1, 1-9.

Roeckner, E. et al., 2003: The atmospheric general circulation model ECHAM5. Part ii: Simulated climatology and comparison with observations. Tech. Rep. 349, MPI-M, Hamburg, Germany.

Seifert, A. and K. D. Beheng, 2001: A double-moment parameterization for simulating autoconversion, accretion and self collection. Atmos. Res., 59-60, $265-281$.

Stevens, B., 2007: On the growth of layers of nonprecipitating cumulus convection. J. Atmos. Sci., 64, 2916-2931.

Stevens, B., C. H. Moeng, and P. P. Sullivan, 1999: Large-eddy simulation of radiatively driven convection: sensivities to the representation of small scales. J. Atmos. Sci., 56, 3963-3984. 
Stevens, B. et al., 2005: Evaluation of large-eddy simulations via observations of nocturnal marine stratocumulus. Mon. Wea. Rev., 133, 1443-1462.

Tiedke, M., 1989: A comprehensive mass flux scheme for cumulus param- eterization in large-scale models. Mon. Wea. Rev., 117, 1779-1800.

Vintzileos, A. and R. Sadourny, 1997: A general interface between an atmospheric general circulation model and underlying ocean and land surface models: delocalized physics scheme. Mon. Wea. Rev., 125, 926-941.

Wan, H., 2009: Developing and testing a hydrostatic atmospheric dynamical core on triangular grids. Ph.D. thesis, Int. Max Planck Res. School.

Williamson, D. L., 1999: Convergence of atmospheric simulations with increasing horizontal resolution and fixed forcing scales. Tellus, 51A, 663-673.

Williamson, D. L., 2008: Convergence of aqua-planet simulations with increasing resolution in the community atmospheric model, version 3 . Tellus, $60 \mathrm{~A}$, 848-862.

Williamson, D. L. and J. G. Olson, 2003: Dependence of aqua-planet simulations on time step. Q. J. R. Meteorol. Soc., 129, 2049-2064. 